\title{
1 Recurrent processing drives experience-dependent plasticity for perceptual decisions
}

$3 \mathrm{Ke} \mathrm{Jia}^{1}$, Elisa Zamboni ${ }^{1}$, Valentin Kemper ${ }^{3}$, Catarina Rua ${ }^{2}$, Nuno Reis Goncalves ${ }^{1}$, Adrian Ka

4 Tsun $\mathrm{Ng}^{1,4}$ Christopher T. Rodgers ${ }^{2}$, Guy Williams ${ }^{2}$, Rainer Goebel ${ }^{3}$ and Zoe Kourtzi ${ }^{1, *}$

$6 \quad{ }^{1}$ Department of Psychology, University of Cambridge, Cambridge, CB2 3EB, UK.

$7{ }^{2}$ Wolfson Brain Imaging Centre, Department of Clinical Neurosciences, University of

8 Cambridge, Cambridge, CB2 0QQ, UK.

$9{ }^{3}$ Department of Cognitive Neuroscience, Faculty of Psychology and Neuroscience, Maastricht

10 University, Maastricht, 6200 MD, The Netherlands.

$11{ }^{4}$ Department of Industrial and Manufacturing Systems Engineering, The University of Hong

12 Kong, Hong Kong SAR, China.

13

14

15 Correspondence:

16 Zoe Kourtzi

17 Department of Psychology

18 University of Cambridge

19 Cambridge, UK

20 Email: zk240@cam.ac.uk 


\section{Abstract}

23 Learning and experience are critical for translating ambiguous sensory information from our

24 environments to perceptual decisions. Yet, evidence on how training molds the adult human

25 brain remains controversial, as fMRI at standard resolution does not allow us to discern the finer-

26 scale mechanisms that underlie sensory plasticity. Here, we combine ultra-high field (7T)

27 functional imaging at sub-millimetre resolution with orientation discrimination training to

28 interrogate experience-dependent plasticity across cortical depths. Our results provide evidence

29 for recurrent plasticity, by contrast to sensory encoding vs. feedback mechanisms. We

30 demonstrate that learning alters orientation-specific representations in superficial rather than

31 middle V1 layers, suggesting changes in read-out rather than input signals. Further, learning

32 increases feedforward rather than feedback layer-to-layer connectivity in occipito-parietal

33 regions, suggesting that sensory plasticity gates perceptual decisions. Our findings propose finer-

34 scale plasticity mechanisms that re-weight sensory signals to inform improved decisions,

35 bridging the gap between micro- and macro- circuits of experience-dependent plasticity.

\section{Keywords}

38 ultra-high field brain imaging, learning, experience-dependent plasticity, perceptual decisions, 39 visual cortex 


\section{Introduction}

41 Understanding the world around us depends on the brain resolving ambiguous information from

42 our senses to inform our decisions and actions. The brain learns to interpret sensory signals by

43 using past experience to optimize perceptual judgments. Although this experience-dependent

44 brain plasticity is most evident during development, training in adulthood is shown to improve

45 visual recognition skills and alter the brain's function and connectivity ${ }^{1-4}$. Yet, evidence on how

46 practice molds the adult human brain and results in improved decisions remains controversial.

47 The neural computations that mediate experience-dependent plasticity are highly debated

48 (e.g. ${ }^{1,5,6}$ ). Results from behavioral training studies focusing on visual discrimination tasks (i.e.

49 perceptual learning tasks) have long been understood to suggest that plasticity occurs at early

50 stages of sensory processing (i.e. primary visual cortex), as learning was shown to be specific to

51 the trained stimulus features (e.g. ${ }^{7,8}$ ). Neurophysiological recordings ${ }^{9,10}$ and human imaging data

$522^{11-13}$ support this early neural locus hypothesis by showing that training changes neural responses

53 in primary visual cortex, implying that learning alters stimulus encoding. By contrast, other

54 studies have shown that learning alters processing in higher visual areas ${ }^{14,15}$ and regions involved

55 in decision-making ${ }^{6,16}$. This suggests that learning changes the read-out of sensory information ${ }^{5}$

56 from higher cortical areas rather than sensory encoding in visual cortex, with changes in activity

57 in early visual cortex reflecting feedback processes ${ }^{17}$.

58 Here, we capitalize on recent advances in brain imaging technology (i.e. Ultra-High Field:

59 UHF imaging) that allow us to interrogate brain computations at a finer scale than that afforded

60 by standard fMRI techniques ${ }^{18}$. UHF imaging affords the sub-millimetre resolution necessary to

61 examine fMRI signals across cortical laminar layers that are known to be associated with

62 dissociable brain computations (Figure 1). In particular, sensory input is known to enter the 
63 cortex from the thalamus at the level of the middle layer (layer 4) and output information is fed

64 forward from the superficial layers (layer 2/3), while feedback information is exchanged

65 primarily between deeper (layer 5/6) and superficial layers ${ }^{19-22}$. Further, horizontal connections

66 across V1 columns are known to predominantly terminate in superficial layers ${ }^{23,24}$ and suggested

67 to support recurrent processing within visual cortex ${ }^{25,26}$. Neurophysiological studies have shown

68 that this micro-circuit is involved in a range of visual recognition ${ }^{26}$ and attention ${ }^{27}$ tasks. Recent $^{2}$

69 laminar fMRI studies provide evidence for the involvement of this circuit in the context of

$70 \quad$ sensory processing ${ }^{28}$ and visual attention ${ }^{29}$.

71

- Feedforward

- Recurrent

- Feedback

\section{Figure 1. Laminar brain circuits.}

74 Schematic representation of the hypotheses tested, training may modify: a) feedforward processing (blue

75 arrows) between LGN and $\mathrm{V} 1$; b) recurrent processing via horizontal connections within the visual cortex

76 (horizontal connections (indicated by orange arrows) between excitatory (open circles) and inhibitory

77 (filled circles) neurons adapted from Teich et al. (2003) and Schwabe et al. (2005)); c) feedback

78 processing (green arrows) between V1 and higher areas (i.e., V2, V3, V4, IPS) based on known 79 anatomical circuits. 
We combine 7T laminar fMRI (i.e. before and after training) with training on an orientation

82 discrimination task, to test whether learning modifies: a) encoding of sensory input in middle V1

83 layers, b) recurrent processing in superficial layers that alters the sensory read-out, c) feedback

84 processing in deeper or superficial V1 layers from higher decision-related regions (i.e. intra-

85 parietal cortex, IPS). Using multi-voxel pattern classification analysis (MVPA) across cortical

86 depths, we demonstrate learning-dependent changes in visual representations that are specific to

87 superficial rather than middle or deeper V1 layers, suggesting that learning modifies read-out

88 rather than input signals in visual cortex. Further, we show enhanced feedforward connectivity

89 between superficial layers in visual cortex and middle layers of posterior parietal cortex, rather

90 than feedback connectivity between deeper layers in these areas. Our findings offer an alternate

91 proposal to the ongoing debate focusing on encoding vs. feedback mechanisms of experience-

92 dependent plasticity: learning is implemented by recurrent computations that alter read-out rather

93 than sensory encoding in visual cortex and feedforward processing from sensory to decision

94 related areas.

\section{Results}

\section{Learning-dependent changes in perceptual discrimination}

98 We trained participants $(\mathrm{N}=15$; data from two participants were excluded due to excessive head

99 movement and technical issues during acquisition) on an orientation discrimination task ${ }^{9,12}$ for

100 five consecutive days and tested their performance on the same task during fMRI scanning

101 before and after training (Figure 2A\&B). Participants' discrimination performance improved

102 during training (Figure 2C), as indicated by a significant decrease in threshold $(\sim 79.4 \%)$

103 performance (paired t-test, mean threshold on day 1 vs. day 5: $\mathrm{t}(12)=8.108, p<0.001$ ). 
To determine the specificity of this learning, we measured participants' discrimination

105 threshold for two different orientations (i.e., trained vs. untrained orientations that corresponded

106 to $55^{\circ}$ or $125^{\circ}$ ) at two different locations (i.e., trained vs. untrained location that corresponded to

107 the left or right visual field) before and after training. Our results showed that behavioral

108 improvement due to training was stronger for the trained orientation and location (Figure 2D,

109 Figure S1), consistent with previous studies ${ }^{8}$ showing specificity to the trained stimulus features.

110 In particular, we observed a significant orientation $\times$ location $\times$ session interaction (repeated

111 measures ANOVA, $\mathrm{F}(1,12)=11.858, p=0.005)$ and a significant orientation $\times$ session

112 interaction $(\mathrm{F}(1,12)=21.551, p=0.001)$ at the trained, but not the untrained $(\mathrm{F}(1,12)=3.093, p$

$113=0.104)$ location. As suggested by recent work ${ }^{7}$, it is likely that this learning specificity is due to

114 prolonged training near threshold (i.e. employing a single staircase ${ }^{30}$ ), in contrast to supra-

115 threshold training that has been suggested to enhance transfer and higher-level learning ${ }^{31}$. 
A

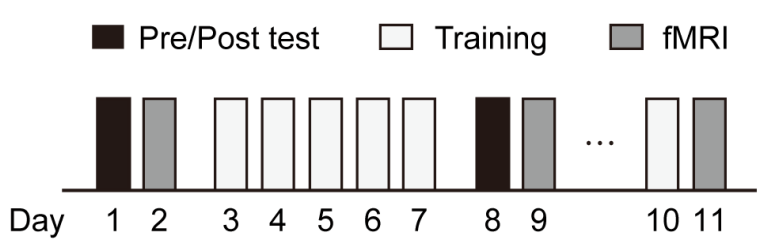

C

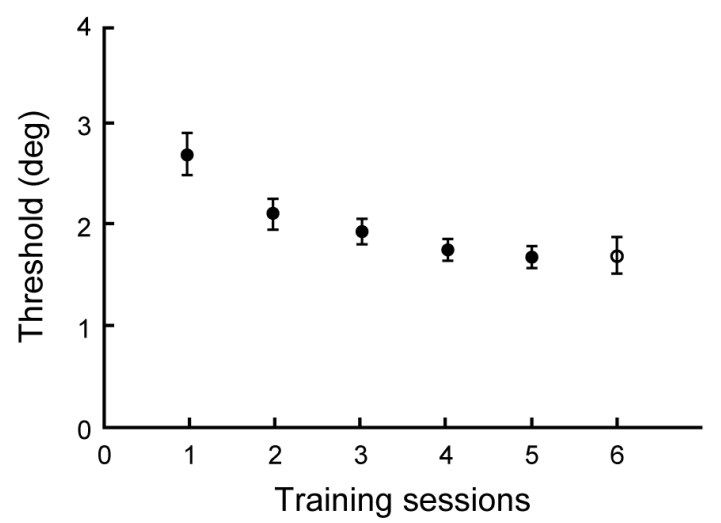

B

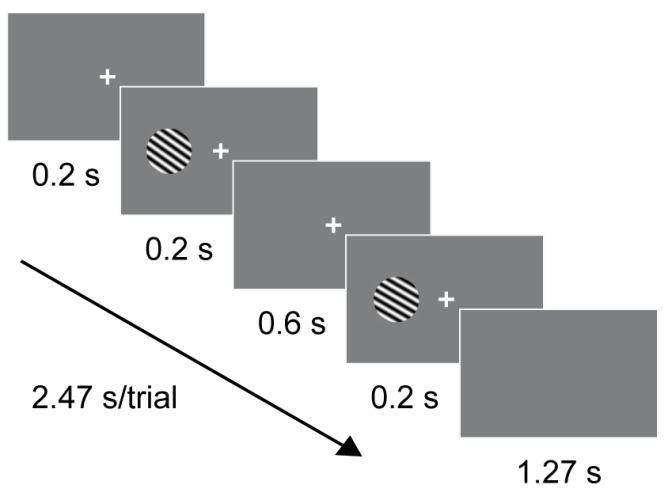

Trained location

Untrained location

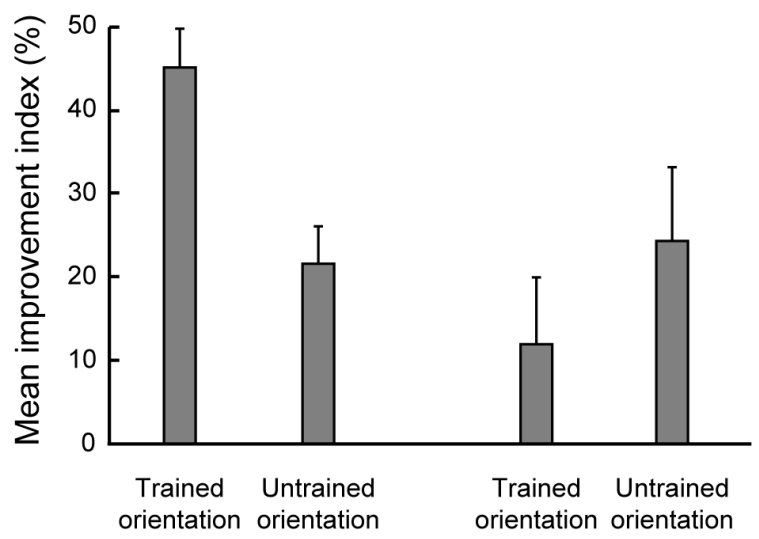

Figure 2. Experimental design, task and behavioral results.

119 (A) Experimental design. Participants were trained on an orientation discrimination task with feedback for

120 five consecutive days. Before and after training, we measured participant's performance on the same task

121 without feedback in the lab and during scanning. (B) Orientation discrimination task. For each trial,

122 participants were asked to report whether the second grating was tilted clockwise or counterclockwise

123 relative to the first grating. (C) Mean performance across participants at $79.4 \%$ threshold for the training

124 (filled circles) and the control (open circle) sessions. (D) Mean improvement index (MPI = (pre-test

125 threshold - post-test threshold) / pre-test threshold $\times 100 \%$ ) showed learning specificity for the trained

126 compared to the untrained orientation presented at the trained vs. untrained location. A two-way repeated

127 measures ANOVA on MPI (orientation $\times$ location) showed a significant interaction $(\mathrm{F}(1,12)=14.847, p$

$128=0.002$ ). Post-hoc comparisons showed significantly higher improvement for the trained than the 
untrained $(\mathrm{t}(12)=5.564, p<0.001)$ orientation at the trained location. In contrast, no significant

130 differences were observed between the trained and the untrained $(\mathrm{t}(12)=-1.608, p=0.134)$ orientations at

131 the untrained location. Error bars indicate standard error of the mean across participants.

\section{Learning-dependent changes across cortical depth in the visual cortex}

134 To test whether learning alters orientation representations across cortical depth in the visual

135 cortex, we segmented the visual areas and assigned voxels to three layers (superficial, middle,

136 deeper) using an equi-volume approach (see Methods, Anatomical data analyses for details;

137 Figure 3A-D). We used MVPA to discern orientation-specific fMRI signals and test for

138 differences in these signals before vs. after training across layers. In particular, we tested whether

139 linear classifiers that were trained on fMRI signals from multi-voxel patterns in different V1

140 layers (superficial, middle, deeper) discriminated between: a) trained $\left(55^{\circ}\right.$ or $\left.125^{\circ}\right)$ vs. control

141 (vertical) orientations, b) untrained $\left(125^{\circ}\right.$ or $\left.55^{\circ}\right)$ vs. control (vertical) orientations. Classifying

142 each of the reference orientations $\left(55^{\circ}\right.$ or $\left.125^{\circ}\right)$ from the control (vertical) orientation allowed us

143 to test learning-dependent changes separately for the trained vs. untrained orientations. We

144 hypothesized that higher MVPA accuracy after training for the trained vs. control orientation

145 classification than the untrained vs. control orientation classification would indicate orientation-

146 specific learning-dependent plasticity. Note that, as both the trained and untrained orientation

147 differed equally from the control orientation $\left(\sim 55^{\circ}\right)$, accuracy differences between these

148 classification tasks could not be attributed to stimulus differences. 
A

B

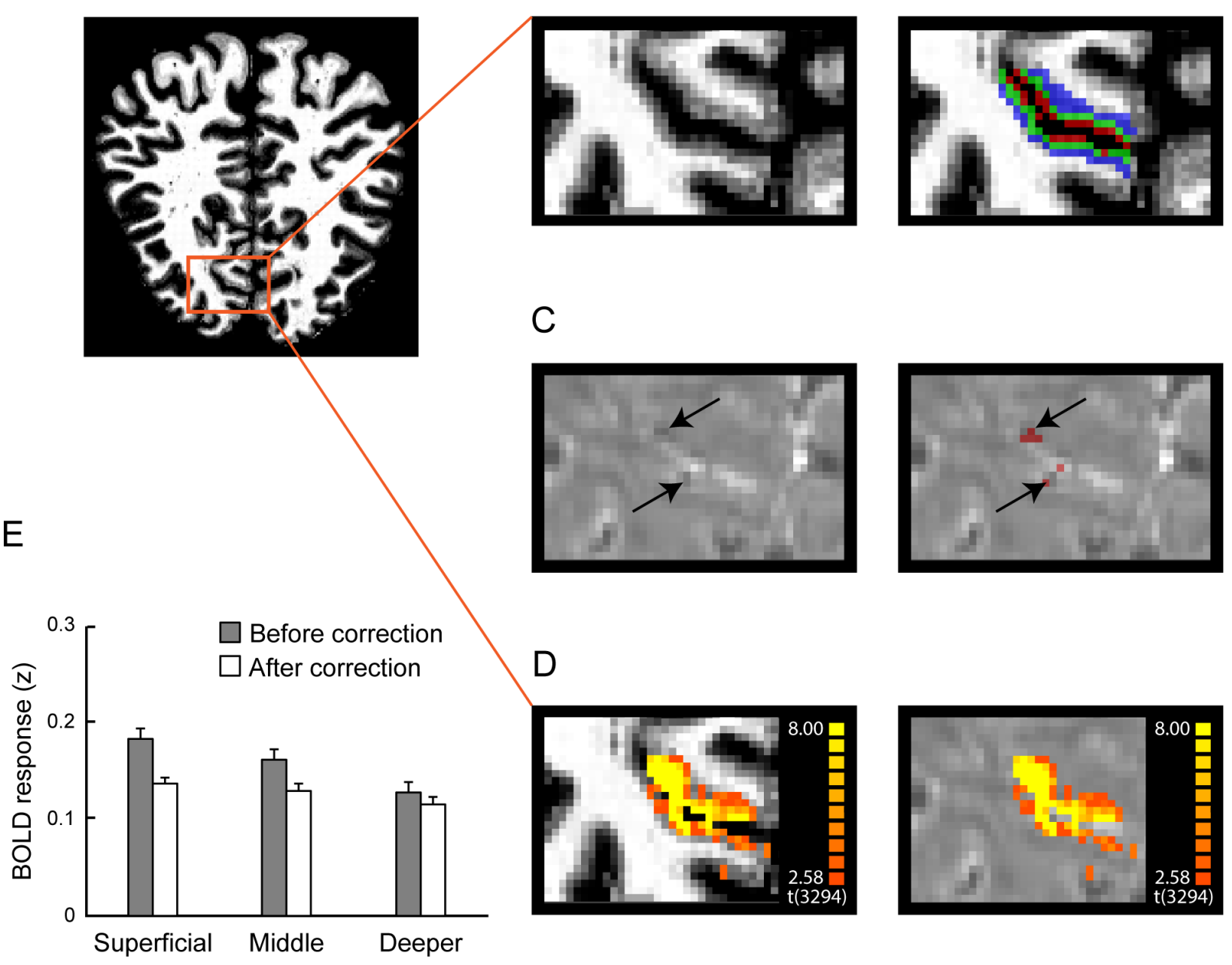

151 Figure 3. fMRI layer definition and vascular correction.

152 (A) Coronal view of the anatomical image of a sample participant. Red insert indicates region of interest

153 in visual cortex. (B) Layers definition map overlaid on an anatomical image (blue: deeper layers, green:

154 middle layers, and red: superficial layers). (C) Voxels confounded by vasculature-related effects

155 (highlighted by arrows and in red) overlaid on functional images. (D) BOLD activation map (stimulus vs.

156 fixation) overlaid on the anatomical (left panel) and functional data (right panel). (E) Mean normalized

157 BOLD in V1 before and after correction for vasculature-related effects, showing reduced superficial bias

158 after correction. Error bars indicate standard error of the mean across participants. We observed

159 significant interactions (pre-test session: $\mathrm{F}(2,24)=50.961, p<0.001$, post-test session: $\mathrm{F}(2,24)=36.887$, 
$p<0.001$ ) between layer (superficial, middle, deeper) and BOLD signal (before vs. after correction). The

161 stronger BOLD decrease in upper (i.e. superficial, middle) than deeper layers after correction suggests

162 that our approach for correcting vasculature-related effects controlled substantially for the superficial bias. trained orientation in superficial rather than middle or deeper layers in V1 (Figure 4, Figure 5A).

166 In particular, a three-way repeated measures ANOVA (orientation $\times$ session $\times$ layer) on the

167 MVPA accuracy showed a significant three-way interaction $(\mathrm{F}(2,24)=4.244, p=0.026)$. Two-

168 way repeated measures ANOVAs (orientation $\times$ session) showed a significant interaction in 169 superficial V1 layers $(\mathrm{F}(1,12)=12.223, p=0.004)$, but not in middle $(\mathrm{F}(1,12)=0.012, p=$ 170 0.913), nor deeper $(\mathrm{F}(1,12)=0.446, p=0.517)$ layers. Further, we observed enhanced

171 discriminability (i.e. MVPA accuracy) for the trained orientation in superficial $(\mathrm{t}(12)=-2.665, p$

$172=0.021)$, but not middle $(\mathrm{t}(12)=-0.783, p=0.449)$, nor deeper $(\mathrm{t}(12)=-0.489, p=0.633)$ layers.

173 In contrast, we did not observe any significant learning-dependent changes for orientations

174 presented in the untrained location. In particular, there was no significant three-way interaction

175 (orientation $\times$ session $\times$ layer, $\mathrm{F}(2,24)=0.603, p=0.555)$ nor any significant orientation $\times$

176 session interactions across V1 layers (superficial layers: $\mathrm{F}(1,12)=0.053, p=0.821$; middle

177 layers: $\mathrm{F}(1,12)=0.538, p=0.478$; deeper layers: $\mathrm{F}(1,12)=2.211, p=0.163)$. Taken together,

178 our results showed that learning-dependent changes in orientation representations in superficial

179 V1 layers were specific to the trained orientation and location. It is unlikely that these learning-

180 dependent changes in visual representations were due to differences in attention related to task

181 difficulty before vs. after training, as participant performance was matched across scanning 182 sessions. 


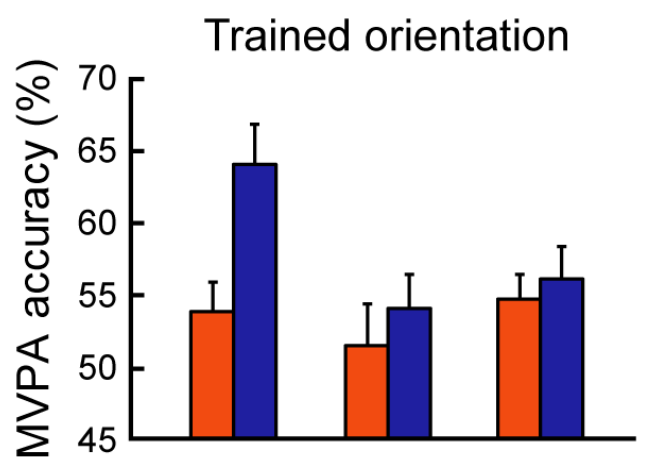

Superficial Middle Deeper
Untrained orientation

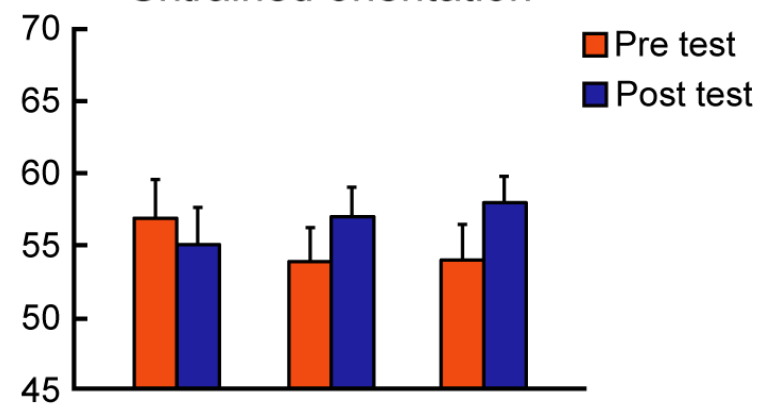

Superficial Middle Deeper
185

186

187

188

189

190

191

192

\section{Complementary and control analyses}

194 To further validate our results and control for potential confounds we conducted the following 195 additional analyses.

First, it has been shown that the overall BOLD signal as measured by GE-EPI is higher at the cortical surface due to vascular contributions ${ }^{32}$ resulting in loss of spatial specificity ${ }^{33}$. Here, we combined several approaches to reduce this superficial bias by removing voxels with low temporal signal to noise ratio and high t-statistic for stimulation contrast (see Methods, correcting for vascular effects for details). We then z-scored each voxel's time course to account

201 for possible differences in signal strength and variance due to thermal or physiological noise 202 across layers while preserving differences between conditions ${ }^{29}$. These corrections resulted in 
203 similar BOLD magnitude and multi-voxel pattern classification accuracy before training across

204 layers (Figure 3E), suggesting that our approach for correcting vasculature-related effects

205 controlled substantially for the superficial bias. In particular, consistent with previous studies ${ }^{34}$

206 showing reduced superficial bias for MVPA classification measures, we did not observe any

207 significant differences in MVPA accuracy between trained and untrained orientations before

208 training $($ e.g. orientation $\times$ location $\times$ layer interaction: $\mathrm{F}(2,24)=0.891, p=0.423$; main effect

209 of layer: $\mathrm{F}(2,24)=0.287, p=0.753)$. Thus, it is unlikely that our MVPA results after vasculature

210 correction were significantly confounded by the superficial bias.

211 Second, we applied a spatial regression approach ${ }^{35,36}$ to control for signal contribution from

212 draining veins. In particular, intra-cortical veins running perpendicular to the cortical surface are

213 known to drain blood from deeper layers of the cortex to larger pial veins situated along the grey

214 matter surface, resulting in loss of spatial specificity and intra-layer BOLD signal contamination.

215 To unmix the signal from adjacent layers, for each voxel in the superficial layers, we found the

216 nearest neighbor in the middle layer. We regressed out the mean time course of these voxels

217 assigned to middle layers from the time course of voxels assigned to superficial layers. MVPA

218 analysis following this correction showed a significant interaction between orientation and

219 session $(\mathrm{F}(1,12)=14.357, p=0.003$, Figure S2A), consistent with learning-dependent changes

220 in superficial layers. Further, we observed enhanced discriminability in superficial layers after

221 correction for the trained orientation $(\mathrm{t}(12)=-2.292, p=0.041)$, but not the untrained orientation

$222(\mathrm{t}(12)=0.246, p=0.810)$. Learning-dependent changes in superficial V1 layers remained

223 significant after these corrections, suggesting that our results are unlikely to be significantly

224 confounded by vasculature-related artifacts. 
Third, comparing mean normalized fMRI responses (Figure S3) across orientations and

226 sessions did not show any significant results (three-way interaction (orientation $\times$ session $\times$

227 layer): $\mathrm{F}(4,48)=1.259, p=0.299$, two-way interaction (orientation $\times$ session) in the superficial

228 layers: $\mathrm{F}(2,24)=0.814, p=0.455$, middle layers: $\mathrm{F}(2,24)=0.934, p=0.407$ and deeper layers:

$229 \mathrm{~F}(2,24)=0.389, p=0.682)$, suggesting that the learning-dependent effects we observed reflect

230 changes in orientation-specific representations across voxel patterns rather than mean univariate

231 fMRI responses.

232 Fourth, we corroborated our results using a correlation-based pattern analysis ${ }^{37}$ that showed

233 learning-dependent changes in superficial V1 layers for the trained compared to the untrained

234 orientation (Figure S2B). Specifically, Fisher z comparisons showed a significant orientation $\times$

235 session interaction in superficial V1 layers $(\mathrm{F}(1,12)=6.069, p=0.030)$, but not in middle

$236(\mathrm{~F}(1,12)=2.382, p=0.149)$ nor deeper layers $(\mathrm{F}(1,12)=1.227, p=0.290)$.

237 Taken together, these results demonstrate enhanced orientation-specific representations in 238 superficial rather than middle or deeper V1 layers after training, suggesting that learning alters

239 read-out rather than input processing in V1. Similar learning-dependent effects with stronger

240 learning-dependent changes in superficial layers were observed across visual areas (V1, V2, V3,

241 V4, Figure S4). In particular, a repeated measures ANOVA showed no significant ROI x

242 orientation $\mathrm{x}$ session $\mathrm{x}$ layer interaction $(\mathrm{F}(2,24)=1.459, p=0.252)$, but a significant orientation

$243 \mathrm{x}$ session $\mathrm{x}$ layer interaction $(\mathrm{F}(2,24)=5.305, p=0.012)$, suggesting similar orientation-specific

244 learning effects in superficial layers across visual areas. 


\section{Learning-dependent changes independent of task-context in the visual cortex}

249 Our results showed learning-dependent changes in orientation-specific representations when

250 participants performed a fine orientation discrimination task. To test whether the task performed

251 by the participants is critical for this orientation-specific plasticity, we tested a subset of

252 participants $(n=8)$ in a second post-training fMRI session while performing a control task (i.e.

253 contrast change detection task) on identical stimuli to those presented to the participants during

254 the post-training fMRI session. Before this additional scanning session, we conducted behavioral

255 tests to ensure that perceptual improvement was retained. We observed that threshold

256 performance $\left(\right.$ Mean $\left.=1.78^{\circ}, \mathrm{SD}=0.43^{\circ}\right)$ did not differ significantly from the mean threshold of

257 training day $5\left(\right.$ Mean $=1.69^{\circ}, \mathrm{SD}=0.37^{\circ}$, paired t-test, $\left.\mathrm{t}(7)=-1.553, p=0.164\right)$. Further, we

258 matched task difficulty in the contrast change detection task $(\sim 79.4 \%)$ to performance in the

259 orientation discrimination task during the post-training fMRI session to ensure that the two post-

260 training scanning sessions did not differ in task difficulty.

261 MVPA analysis across cortical layers in V1 showed that the learning-dependent changes we

262 observed in orientation-specific representations in superficial layers were maintained when

263 participants performed the control task. In particular, a two-way repeated measures ANOVA

264 (orientation $\times$ task) on the MVPA accuracy showed a significant main effect of orientation $(\mathrm{F}(1,7)$

$265=19.140, p=0.003)$ in superficial V1 layers (Figure 5B). Neither the main effect of task $(\mathrm{F}(1,7)$

$266=2.608, p=0.150)$ nor the interaction effect $(\mathrm{F}(1,7)=0.082, p=0.783)$ were significant. We did

267 not find any significant main or interaction effects in the deeper or middle layers of V1 (all ps >

268 0.071). These results suggest that learning-dependent changes in orientation-specific

269 representations in superficial V1 layers are independent of task context, consistent with previous 
270 neurophysiology results ${ }^{9}$ showing learning-dependent changes in neural tuning in primary visual

271 cortex during a fixation task.

272

A
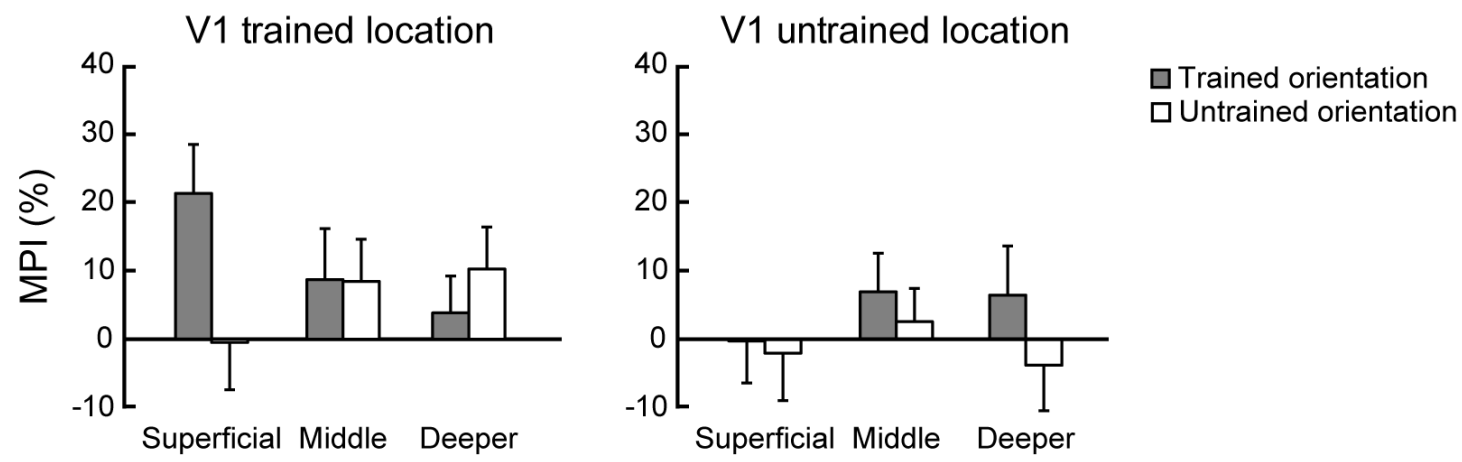

B

273

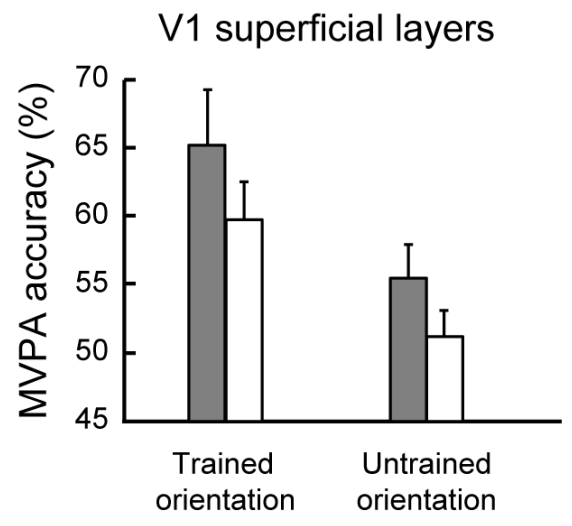

$\square$ Post test

$\square$ Control

274 Figure 5. Learning-dependent changes in V1.

275 (A) Mean improvement index (MPI: (post-test accuracy - pre-test accuracy) $/$ pre-test accuracy $\times 100 \%$ )

276 for the trained and untrained orientations across V1 layers. The left panel shows significantly higher MPI

277 for the trained than the untrained orientation at the trained location in superficial V1 layers, as indicated

278 by significantly higher MPI for the trained than untrained orientation in the superficial layers $(\mathrm{t}(12)=$

$2793.218, p=0.007)$. The right panel shows no significant differences in MPI across layers for orientations

280 presented at the untrained location. In particular, there was no significant differences in MPI for trained vs.

281 untrained orientations in the superficial layers $(\mathrm{t}(12)=0.396, p=0.699)$. (B) MVPA accuracy for the 
trained and untrained orientation at the trained location after training (post-test) compared to the control experiment in superficial layers of V1. Error bars indicate standard error of the mean across participants.

\section{Learning-dependent changes in intraparietal cortex}

We next considered learning-dependent changes in decision-making related areas ${ }^{6,38}$ outside the

287 visual cortex. In particular, we focused on IPS1 and IPS2 that have been implicated in perceptual

288 decision making ${ }^{38}$. Using MVPA, we tested for learning-dependent changes in orientation

289 representations across cortical depth. Figure 6 shows learning-dependent changes for the trained

290 compared to the untrained orientation (i.e. increased MVPA accuracy for the trained orientation

291 in the trained location) in middle rather than superficial or deeper layers. In particular, we

292 observed a significant orientation $\times$ session interaction in middle layers of IPS $(F(1,12)=6.324$,

$293 p=0.027)$, but not in superficial $(\mathrm{F}(1,12)=0.502, p=0.492)$, nor deeper $(\mathrm{F}(1,12)=2.452, p=$

294 0.143) layers. Further, we observed significantly increased MVPA accuracy after training for the

295 trained orientation in the middle $(\mathrm{t}(12)=-3.432, p=0.005)$, but not superficial $(\mathrm{t}(12)=0.392, p$

$296=0.702)$, nor deeper $(\mathrm{t}(12)=-1.137, p=0.278)$ layers, suggesting enhanced discriminability for

297 the trained orientation in middle layers. In contrast, we did not observe any significant learning-

298 dependent changes for the untrained location. In particular, there was no significant orientation $\times$

299 session interactions across IPS layers (superficial layers: $\mathrm{F}(1,12)=0.136, p=0.718$; middle

300 layers: $\mathrm{F}(1,12)=1.053, p=0.325$; deeper layers: $\mathrm{F}(1,12)=1.628, p=0.226$ ). Comparing

301 learning-dependent changes in visual and posterior parietal cortex showed dissociable results.

302 That is, we observed learning-dependent changes in superficial layers of visual cortex, while

303 middle layers of posterior parietal cortex, as indicated by a significant ROI (V1, IPS) x session x

304 layer interaction for the trained orientation (repeated measures ANOVA, $\mathrm{F}(2,24)=5.872, p=$ 


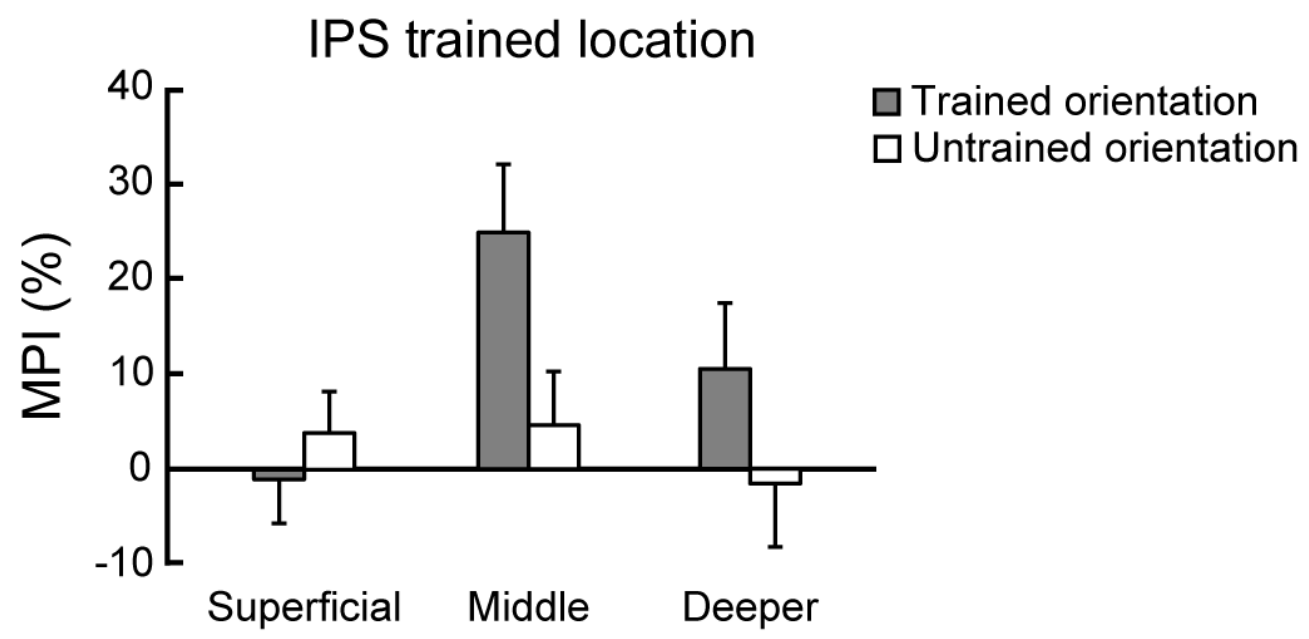

309 Figure 6. Learning-dependent changes in IPS.

310 Mean improvement index across IPS layers for the trained and untrained orientations presented at the

311 trained location. The results showed significantly higher MPI for the trained than the untrained orientation

312 at the trained location in the middle IPS layers $(\mathrm{t}(12)=2.861, p=0.014)$, but not in superficial $(\mathrm{t}(12)=$ -

$3130.695, p=0.500)$, nor deeper $(\mathrm{t}(12)=1.382, p=0.192)$ layers. Error bars indicate standard error of the

314 mean across participants.

\section{Informational connectivity analysis}

317 Our results so far showed learning-dependent changes in orientation-specific representations in

318 the superficial layers of V1 and middle layers of IPS, suggesting that learning modifies read-out

319 rather than input signals in visual cortex, while input signals in posterior parietal cortex. Based

320 on these results, we asked whether learning enhances functional connectivity between visual and

321 posterior parietal cortex. To test this hypothesis, we employed an informational connectivity 322 analysis $^{36}$ and tested whether V1 and IPS shared synchronous discriminability of multi-voxel 
323 patterns that changed with training. Consistent with previous studies ${ }^{19,20,26}$, we contrasted two

324 possible functional connectivity mechanisms: a) feedforward learning, as indicated by changes in

325 connectivity between superficial V1 layers and middle IPS layers, b) feedback learning, as

326 indicated by changes in connectivity between V1 deeper layers and IPS deeper layers. We did

327 not test functional connectivity between V1 superficial layers and deeper layers of higher areas,

328 as it is known to relate to both feedback and feedforward processing (Figure 1).

329 Following previous studies employing an informational connectivity analysis ${ }^{36}$, we

330 interrogated the MVPA classifiers and extracted the distance from the hyperplane for the mean

331 pattern signal per block. For each layer per ROI we generated a time course of distance values

332 across blocks, regressed out the distance from other layers within the ROI and calculated the

333 partial Spearman correlation between V1 and IPS layers across blocks (Figure 7A). Our results

334 showed enhanced feedforward compared to feedback connectivity between V1 and IPS after

335 training. A repeated measures ANOVA (Fisher's z) showed a significant pathway (feedforward,

336 feedback) $x$ orientation (trained vs. untrained) $x$ session (pre-test, post-test) interaction $(\mathrm{F}(1,12)=$

$3378.912, p=0.011$, Figure 7B).

338 In particular, we observed enhanced feedforward connectivity between superficial V1 layers

339 and middle IPS layers after training. A repeated measures ANOVA (Fisher's z) showed a

340 significant orientation $\times$ session interaction $(\mathrm{F}(1,12)=5.771, p=0.033)$, but no significant main

341 effect of orientation $(\mathrm{F}(1,12)=1.218, p=0.291)$ nor $\operatorname{session}(\mathrm{F}(1,12)=2.326, p=0.153)$. Post-

342 hoc comparisons showed enhanced connectivity for the trained orientation $(\mathrm{t}(12)=-2.599, p=$

$3430.023)$, but not the untrained orientation $(\mathrm{t}(12)=1.560, p=0.145)$. In contrast, we did not

344 observe any significant learning-dependent changes in feedback connectivity between deeper V1

345 and deeper IPS layers (orientation $\times$ session interaction: $\mathrm{F}(1,12)=0.587, p=0.458$, main effect 
of session: $\mathrm{F}(1,12)=0.299, p=0.595$, main effect of orientation: $\mathrm{F}(1,12)=1.223, p=0.290)$.

347 This enhanced connectivity between superficial layers of V1 and middle layers of IPS after

348 training suggests that training alters feedforward information processing from sensory to

349 decision-related areas to support learning-dependent improvement in the fine orientation

350 discrimination task.

A
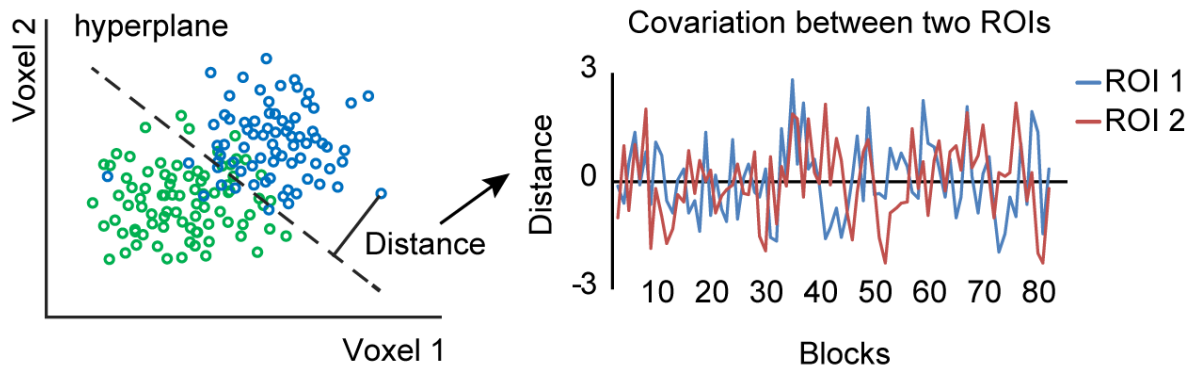

352

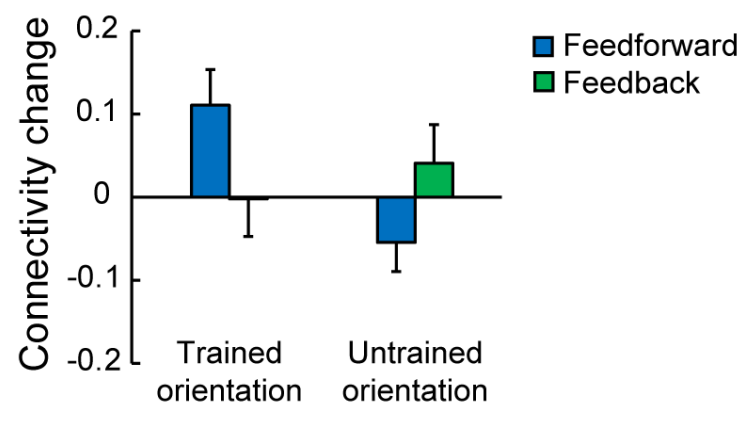

353 Figure 7. Informational connectivity analysis.

354 (A) Schematic illustration of the procedure followed for the MVPA-based functional connectivity 355 analysis. For each ROI and block, the distance to the hyperplane was used as an index of pattern 356 discriminability (left panel). Spearman correlation was used to calculate covariance between two ROIs 357 (right panel). (B) Learning-dependent changes (Fisher's z post- minus pre-test) in functional connectivity between superficial V1 layers and middle IPS layers (feedforward connectivity) and between deeper V1 
and IPS layers (feedback connectivity) for the trained and untrained orientations. Error bars indicate standard error of the mean across participants.

362 Discussion

363 Despite the key role of learning in optimizing perceptual decisions, an ability known as

364 perceptual learning, we lack a mechanistic account of how training molds the adult human brain.

365 Evidence on experience-dependent plasticity mechanisms remains controversial with some

366 studies suggesting that learning modifies sensory encoding, while others proposing top-down

367 influences via feedback ${ }^{5,39,40}$. Conventional brain imaging techniques have been unable to

368 differentiate these possibilities due to limited spatial resolution ${ }^{18}$. Here, we capitalize on the sub-

369 millimeter resolution of 7T laminar fMRI to interrogate plasticity mechanisms across cortical

370 depths that are known to be associated with dissociable neural computations. Our results provide

371 evidence for recurrent experience-dependent plasticity mechanisms, offering an alternate

372 proposal for the neural instantiation of perceptual learning, by contrast to the long-standing

373 debate on encoding vs. feedback mechanisms of sensory plasticity.

374 Combining 7T laminar fMRI with MVPA we demonstrate enhanced decoding of

375 orientation-specific representations in superficial rather than middle layers of V1, suggesting that

376 learning modifies read-out rather than input signals in visual cortex ${ }^{26}$. Previous fMRI studies

377 using multi-voxel pattern classification approaches have shown that learning fine feature

378 discriminations increases the discriminability of neural representations ${ }^{12,41}$. Other brain imaging

379 studies $^{11,13,42,43}$ have shown learning-dependent changes in overall BOLD signal in V1. These

380 fMRI results have been interpreted broadly in support of an early neural locus of perceptual

381 learning. However, we still lack a mechanistic account of experience-dependent plasticity in the

382 human brain, as fMRI at standard resolution does not allow us to discern encoding from read-out 
processes. Our layer-specific fMRI results propose that learning alters the processing of read-out

384 signals in superficial V1 layers rather than stimulus encoding in middle layers. It is unlikely that

385 this learning-dependent plasticity in superficial layers relates to stronger orientation selectivity in

386 superficial V1 layers, as recent quantitative measurements show nearly uniform distribution of

387 orientation selectivity across V1 layers than reported in early physiological studies ${ }^{44}$. Further, we

388 found that MVPA accuracy did not differ significantly across layers before training, suggesting

389 that learning-dependent changes in superficial V1 layers could not be due to differences in

390 orientation selectivity across V1 layers.

391 A possible explanation is that learning-dependent changes in superficial layers reflect top-

392 down influences via feedback ${ }^{45,46}$. It is possible that neurons with cell bodies in deeper layers and

393 dendrites projecting to the superficial layers result in learning-dependent changes in BOLD

394 signals in superficial layers ${ }^{20,21}$. However, this interpretation is less likely for three main reasons.

395 First, we did not observe learning-dependent changes in deeper V1 layers that are known to

396 receive long-range feedback. Second, our functional connectivity analysis showed enhanced

397 feedforward rather than feedback connectivity in occipito-parietal circuits. Third, consistent with

398 a previous physiological study ${ }^{9}$, learning-dependent changes in superficial V1 layers were

399 maintained independent of task context; that is, these changes were evident not only when

400 participants performed the orientation discrimination task, but also when they performed a

401 contrast detection task (i.e. control experiment) that does not involve task-related feedback on

402 the trained stimulus dimension (i.e. orientation). It is possible that top-down influences, via

403 feedback connections to superficial layers, play a role in shaping visual feature templates at

404 earlier than later stages of training on the discrimination task ${ }^{47}$. However, interrogating the 
representations of trained features after training reveals learning-dependent changes in

406 superficial layers that are independent of task context.

407 An alternate proposal is that orientation-specific learning-dependent changes in superficial

408 V1 layers are due to recurrent processing related to iso-orientation inhibition ${ }^{48}$; that is, 409 suppression of neurons that are selective for the same orientation across columns. Iso-orientation

410 inhibition is shown to be more pronounced in superficial layers and support orientation tuning

411 via horizontal connections between $\mathrm{V} 1$ columns $^{19,23,24}$. It is possible that iso-orientation

412 suppression supports enhanced neural tuning to the trained orientation in superficial V1 layers,

413 suggesting a recurrent experience-dependent plasticity mechanism that alters orientation-specific

414 representations within visual cortex. These results are consistent with computational models

415 proposing that training sharpens neural tuning by altering recurrent connections (i.e. reducing

416 excitatory or increasing inhibitory connections) near the trained orientation ${ }^{25,49}$ and

417 neurophysiological studies showing changes in orientation tuning due to training in visual cortex

4189,10 . Further horizontal connections in superficial V1 layers have been suggested to support

419 recurrent processing in the context of figure-ground segmentation ${ }^{26}$ and experience-dependent

420 plasticity in the context of contour integration ${ }^{2}$. In particular, boundary detection was evidenced

421 in superficial layers $\sim 30 \mathrm{~ms}$ after the initial visual response in the middle layers ${ }^{26}$. Future studies

422 combining laminar fMRI with EEG in humans have the potential to test whether learning-

423 dependent changes in superficial layers follow early sensory processing in middle layers,

424 revealing the dynamics of recurrent experience-dependent plasticity ${ }^{26,27}$.

425 It is important to note that despite the advances afforded by laminar fMRI, GE-EPI is

426 limited by vasculature contribution to BOLD signals at the cortical surface resulting in loss of

427 spatial specificity ${ }^{33}$. Here, we demonstrate layer-specific learning-dependent changes following 
428 several control analyses for these potential confounds, suggesting that our results are unlikely to

429 be confounded by vasculature-related artefacts. Our results are consistent with previous laminar

430 imaging studies showing BOLD effects in superficial layers in a range of tasks ${ }^{28,29,45,46}$ and could

431 not be simply attributed to differences in attention due to task difficulty, as participant

432 performance was matched across sessions (pre vs. post-training). Future work could exploit

433 recent advances in $\mathrm{CBV}$ imaging using vascular space occupancy (VASO) ${ }^{50}$ to enhance the 434 spatial specificity of laminar imaging in the human brain.

435 Extending beyond the visual cortex, we demonstrate learning-dependent changes in 436 posterior parietal cortex regions (IPS) that have been suggested to play a key role in perceptual

437 decision making ${ }^{6}$. In particular, we observed learning-dependent changes in fMRI activation 438 patterns in middle layers of IPS regions, suggesting that training alters input signals to posterior 439 parietal cortex. These results are consistent with previous neurophysiological ${ }^{6}$ and human brain 440 imaging studies ${ }^{38}$ that show learning-dependent changes in intraparietal cortex for perceptual 441 decision-making. Our findings provide new insights in understanding the finer scale circuit that 442 mediates experience-dependent plasticity across human brain systems, involving both sensory 443 and decision-related areas ${ }^{51}$.

444 Further, we interrogated learning-dependent changes in functional connectivity ${ }^{38,52}$. We 445 tested whether training alters the functional connectivity within this circuit at the finer scale of 446 layer-to-layer interactions. Computational approaches have proposed that training strengthens the 447 connections between the most informative neurons in sensory areas and decision-related areas 448 via Hebbian learning, resulting in re-weighting of sensory signals in visual cortex ${ }^{5}$. In contrast,

449 the reverse hierarchy theory proposes that learning is implemented by top-down influences to 450 visual processing via long-range feedback from downstream areas ${ }^{17}$. To test these hypotheses, 
451 we interrogated the layer-to-layer functional connectivity between visual and posterior parietal

452 cortex that allows us to compare feedforward vs. feedback processing based on known

453 anatomical connectivity models ${ }^{19,20,53}$. We demonstrate that learning strengthens feedforward

454 connectivity between superficial V1 layers and middle layers of IPS, consistent with previous

455 studies showing that ascending projections of V1 originate predominantly from the superficial

456 layers and ascending projections to IPS mainly terminate in middle layers ${ }^{19,20,26}$. In contrast, we

457 did not find any significant changes in feedback connectivity between deeper V1 and deeper IPS

458 layers, consistent with the lack of significant learning-dependent changes in deeper V1 layers.

459 Taken together, these results suggest that learning fine feature differences is implemented by re-

460 weighting mechanisms of visual plasticity rather than long-range feedback from decision-related

461 to visual areas. Corroborating evidence comes from the results of our control experiment

462 showing that learning-dependent changes in superficial V1 layers were maintained when

463 observers performed a contrast change detection task that does not involve orientation judgments

464 and therefore does not engage decision-related feedback on the trained stimulus dimension.

465 In sum, combining ultra-high field 7T imaging with a classic perceptual learning paradigm,

466 we provide evidence for recurrent mechanisms of experience-dependent plasticity that gate

467 perceptual decision making in the adult human brain. These mechanisms support re-weighting of

468 input signals in the visual cortex that are read-out by posterior parietal cortex to inform improved

469 perceptual decisions due to training. Interrogating experience-dependent plasticity at the finer

470 resolution afforded by UHF imaging using training paradigms that have been extensively tested

471 in both animals and humans, we provide the first insights in bridging the gap between animal

472 studies of micro-circuit plasticity and human fMRI studies of macro-scale network re-

473 organization. Understanding the mechanisms of experience-dependent plasticity in the adult 
bioRxiv preprint doi: https://doi.org/10.1101/2020.04.07.030510; this version posted May 15, 2020. The copyright holder for this preprint (which

was not certified by peer review) is the author/funder, who has granted bioRxiv a license to display the preprint in perpetuity. It is made available under aCC-BY-NC-ND 4.0 International license.

474 brain across scales and species is critical for designing effective training interventions that

475 support lifelong learning and adaptive behavior.

476 


\section{Materials and Methods}

\section{Participants}

479 Fifteen participants (7 females; mean age: 27.87 years and SD: 3.83 years) took part in this study.

480 Data from two participants were excluded from further analysis due to excessive head movement

481 (See MRI data analysis) and technical issues during acquisition. As there are no previous UHF

482 imaging studies on perceptual learning, we determined the sample size based on a previous 3T

483 fMRI study on perceptual learning using an orientation discrimination task $(\mathrm{N}=12)^{12}$. All

484 participants had normal or corrected-to-normal vision, and were right-handed. Participants were

485 naïve to the aim of the study, gave written informed consent and received payment for their

486 participation. The study was approved by the University of Cambridge Ethics committee.

\section{Stimuli and Apparatus}

488 Stimuli comprised oriented sinusoidal gratings that were presented at an eccentricity of $5^{\circ}$, in the

489 left or the right visual field against a uniform gray background. Gratings of random phase had a 490 fixed diameter of $6^{\circ}$, contrast of 0.8 , spatial frequency of 1 cycle/degree. The contrast decreased

491 to zero over the outer $0.5^{\circ}$ radius of the gratings.

492 Experiments were controlled using MATLAB and Psychophysics toolbox 3.0 ${ }^{54,55}$. For the 493 behavioral sessions, stimuli were presented on a 21 -inch CRT monitor $(1600 \times 1200$ pixel 494 resolution, $85 \mathrm{~Hz}$ frame rate) at a distance of $110 \mathrm{~cm}$. Gamma correction was applied to the 495 monitor. For the fMRI scans, stimuli were presented using a projector and a mirror setup $(1920 \times$ 4961080 pixel resolution, $100 \mathrm{~Hz}$ frame rate) at a viewing distance of $110 \mathrm{~cm}$. Angular stimulus size 497 was the same across behavioral and fMRI sessions. 


\section{Experimental design}

501 The study comprised a pre-test (2 sessions, 1 behavioral test, $1 \mathrm{fMRI}$ test), a training ( 5 sessions),

502 a post-test ( 2 sessions, 1 behavioral test, 1 fMRI test) and a control ( 2 sessions: 1 training, 1

503 fMRI test) phase (Figure 2A). All training and post-test sessions were conducted on consecutive 504 days.

505 We employed a two-interval forced choice (2IFC) orientation discrimination task (Figure 506 2B). Each trial began with a fixation cross for $200 \mathrm{~ms}$ followed by the sample and test gratings

507 that were presented sequentially for $200 \mathrm{~ms}$ each and separated by a $600 \mathrm{~ms}$ inter-stimulus

508 interval (ISI). Participants were asked to fixate and report (by key press) within $1270 \mathrm{~ms}$ after the

509 onset of the test grating whether it was tilted clockwise or counter-clockwise relative to the 510 sample stimulus.

511 Participants' performance in the task was measured using a 3-down-1-up staircase with 15

512 reversals converging at $79.4 \%$ performance. The reference orientation for the trained and 513 untrained stimuli was $55^{\circ}$ or $125^{\circ}$. We added a uniformly distributed random jitter within $\pm 5^{\circ}$ to

514 the reference orientation across trials to ensure that participants compared two gratings in each

515 trial, rather than the test grating to a fixed reference orientation. The training reference

516 orientation $\left(55^{\circ}\right.$ vs. $\left.125^{\circ}\right)$ was counterbalanced across participants. Participants were tested with

517 a control orientation $\left(0^{\circ}\right.$, vertical $)$ that differed equally from the trained and untrained orientation

$518\left(55^{\circ}\right.$ or $\left.125^{\circ}\right)$. This allowed us to test orientation-specific pattern changes in fMRI signals due to

519 training (i.e. learning-dependent changes to the trained vs. untrained orientation), by comparing

520 separately the trained vs. the untrained orientations to the control orientation.

521 Behavioral Tests. To familiarize participants with the task before testing, each participant 522 performed a 30-trial practice run (5 trials per condition, i.e. three different reference orientations 
523 at two different locations) using a fixed above-threshold angle difference $\left(8^{\circ}\right)$. For both the pre-

524 and post-training test, participants performed the orientation discrimination task for 12 test

525 staircase runs (2 runs per condition in random order). For each condition, the starting angle

526 difference between sample and test stimulus for the first run was $5^{\circ}$. For the second run, the

527 starting angle difference was determined by the threshold in the preceding run. The

528 discrimination threshold for each condition was the mean threshold across two runs. No feedback

529 was provided to the participants during the test phase.

530 Behavioral Training (5 sessions). We trained participants on the orientation discrimination task

531 (16 staircases per session, $\sim 1 \mathrm{~h}$ ) with gratings presented at the same orientation and location

532 throughout training. The starting angle difference between sample and test stimuli for the first

533 staircase of the first training session was $5^{\circ}$. For the remaining staircases, the starting angle

534 difference was determined by the threshold of the preceding staircase. Training location (i.e., left

535 vs. right visual field) and orientation (i.e., $55^{\circ}$ vs. $125^{\circ}$ ) were counterbalanced across participants.

536 Participants were given auditory error feedback per trial.

537 fMRI sessions. Before and after training in the lab, participants completed 8-10 runs of the

538 orientation discrimination task during scanning. For each participant, we also collected data from

539 an anatomical scan and a retinotopic mapping scan.

540 For the orientation discrimination task, each run started with a fixation block (12.36 sec)

541 followed by one block for each of the six conditions and a fixation block. This sequence of

542 fixation and condition blocks was repeated four times in each run. Each condition block lasted

$54312.36 \mathrm{sec}$ and comprised gratings presented at the trained, untrained or control orientation at one

544 of two locations (trained vs. untrained location). The order of orientations was randomized

545 across the six condition blocks and the stimulus location alternated between blocks. For each 
546 block, participants completed five trials of the orientation discrimination task. The task

547 parameters (i.e. sample and test duration) were the same as for the behavioral tests and no

548 feedback was provided to the participants. The fixed angle difference between sample and test

549 stimuli for each condition was determined by the preceding behavioral session. This allowed us

550 to match task difficulty $(\sim 79.4 \%)$ before and after training.

551 Control experiment. To test the task specificity of the learning effect, eight participants

552 completed an additional training and fMRI session on consecutive days to ensure that learning

553 was maintained during a control task. The procedure for this control-task session was identical to

554 that for the post-training scan, with the exception that participants performed a contrast change

555 detection task. Participants were required to press a key within $1000 \mathrm{~ms}$ of detecting a contrast

556 change on the stimuli. The magnitude of contrast change was estimated for each participant

557 during the anatomical scan to ensure similar task difficulty $(\sim 79.4 \%)$ between the orientation

558 discrimination task and the contrast detection task. The estimated magnitude was fixed and used

559 throughout the control-task fMRI session.

560 MRI data acquisition

561 Imaging data were acquired at the Wolfson Brain Imaging Centre, University of Cambridge, on a

562 Siemens 7T Terra scanner with a 32-channel phased-array head coil (Nova Medical, Inc.,

563 Wilmington, MA, USA). For each participant, anatomical images were acquired using

564 MP2RAGE T1-weighted sequence $\left(\mathrm{TR}=5000 \mathrm{~ms}, \mathrm{TE}=2.56 \mathrm{~ms}, \mathrm{FOV}=208 \times 208 \mathrm{~mm}^{2}\right.$,

565 resolution $0.65 \times 0.65 \times 0.65 \mathrm{~mm}^{3}$, number of slices: 240 , slice orientation: sagittal). Functional

566 scans were acquired using a 2D Gradient Echo, Echo Planar Imaging (GE-EPI) sequence ${ }^{56}(\mathrm{TR}=$

$5672060 \mathrm{~ms}, \mathrm{TE}=26.4 \mathrm{~ms}, \mathrm{FOV}=148 \times 148 \mathrm{~mm}^{2}$, flip angle: $70^{\circ}$, resolution $0.8 \times 0.8 \times 0.8 \mathrm{~mm}^{3}$,

568 number of slices: 56, partial Fourier $=6 / 8$, GRAPPA factor $=3$, Multi-Band factor $=2$, 
569 bandwidth $=1034 \mathrm{~Hz} /$ Pixel, echo spacing $=1.09 \mathrm{~ms})$. The field of view covered occipito-

570 temporal and posterior parietal areas; manual shimming was performed prior to the acquisition of

571 the functional scans.

\section{Behavioral data analysis}

573 Performance was measured by the 3-down-1-up staircase with 15 reversals. The mean angle

574 difference of the last 8 reversals was taken as the threshold of each staircase run. The measured

575 orientation discrimination thresholds were used as the dependent factor. Using a within-subject

576 factorial design, we manipulated three independent factors, the reference orientation (trained and

577 untrained orientation), stimulus location (trained and untrained location) and test session (pre-test,

578 post-test), to evaluate the learning effect and learning specificity. Further, we calculated the

579 mean percent improvement index (MPI, (pre-test threshold - post-test threshold) / pre-test

580 threshold $\times 100 \%)$ ) for each condition. For statistical analysis, we used repeated measures

581 ANOVAs to compare across conditions.

\section{MRI data analysis}

583 Anatomical data analyses. T1-weighted anatomical data was used for coregistration and 3D

584 cortex reconstruction. Grey and white matter segmentation was obtained on the MP2RAGE

585 images using FreeSurfer (http://surfer.nmr.mgh.harvard.edu/) and manually improved for the

586 regions of interest (i.e., V1, V2, V3, V4, and IPS) using ITK-SNAP (www.itksnap.org). The

587 refined segmentation was used to obtain a measurement of cortical thickness. Following previous

588 studies, we assigned voxels to three layers (superficial, middle, deeper) using the equi-volume

$589 \operatorname{approach}^{57,58}$ as implemented in BrainVoyager (Brain Innovation, Maastricht, The Netherlands).

590 This approach has been shown to reduce misclassification of voxels to layers, in particular for

591 regions of interest presenting high curvature. Information from the cortical thickness map and 
592 gradient curvature was used to generate four grids at different cortical depths (ranging from 0:

593 white matter, to 1: grey matter). Mapping of each voxel to a layer was obtained by computing the

594 Euclidean distance of each grey matter voxel to the grids: the two closest grids represent the

595 borders of the layer to which a voxel is assigned (Figure 3B). The anatomical image was aligned

596 to the functional data using the boundary-based registration ${ }^{59}$. We assessed the alignment and

597 manually corrected if necessary.

598 Functional data analyses. The GE-EPI functional data were analysed using BrainVoyager

599 (version 20.6, Brain Innovation, Maastricht, The Netherlands) and custom MATLAB (The

600 MATHWORKS Inc., Natick, MA, USA) code. The first two volumes at the beginning of each

601 run were discarded to ensure that the longitudinal magnetization reached steady state. The

602 functional data were corrected for distortions due to non-zero off-resonance field (at the

603 beginning of each functional run, five volumes with inverted phase encoding direction were

604 acquired and used to estimate a voxel displacement map that was subsequently applied to the

605 functional data using COPE (Correction based on Opposite Phase Encoding, BrainVoyager,

606 Brain Innovation). The distortion-corrected data underwent slice-timing correction, head motion

607 correction (the single band image acquired at the beginning of the first run was used as the

608 reference in the alignment), high-pass temporal filtering (using a GLM with Fourier basis set at 2

609 cycles) and removal of linear trends. We then aligned the functional data across sessions. To

610 validate the alignment, we calculated the mean EPI image of each functional run for each region

611 of interest (ROI) and estimated the spatial correlation between these mean EPI images. We

612 performed manual adjustment of the alignment if the spatial correlation was below 0.85 and

613 excluded data from one participant for whom the alignment could not be improved manually. 
614 Regions of Interest definition. We used the data from the retinotopic mapping scan to identify

615 visual areas based on standard phase-encoding methods. Participants viewed rotating wedges that

616 created travelling waves of neural activity ${ }^{60,61}$. Due to limited coverage during acquisition, area

617 V4 was identified for 8 of the 13 participants included in the analysis. Thus, for further analyses

618 we combined the data from V2, V3 and V4 for each individual participant. Further, we defined

619 regions in the intraparietal sulcus (IPS1, IPS2) given their functional relevance for perceptual

620 learning ${ }^{6}$. Intraparietal regions (IPS1 and IPS2) were defined for each participant based on

621 anatomical templates provided by Benson (https://hub.docker.com/r/nben/occipital atlas/) ${ }^{62}$.

622 This procedure uses the individual participant-based segmentation obtained with FreeSurfer and

623 an anatomical probabilistic template, to estimate the best location for the region of interest (i.e.

624 IPS). Each IPS subregion was subsequently inspected to ensure consistent definition across 625 participants.

626 For each of the visual cortex ROIs, we modelled BOLD signals using a GLM with two 627 regressors (i.e., left vs. right visual field) and included the estimated head motion parameters as 628 nuisance regressors. The resulting $\mathrm{t}$-statistical map was thresholded $(\mathrm{t}=2.58, p=0.01)$ to select 629 voxels within each ROI that responded strongly to the lateralized stimulus presentation, 630 consistent with location specificity in visual cortex. For IPS, we selected voxels that responded 631 to the task irrespective of stimulus location (i.e. task vs. fixation, $\mathrm{t}=1.64, p=0.10$ ).

632 Correcting for vasculature-related effects: Voxel selection within each ROI was further refined

633 by excluding voxels that were confounded by vasculature effects that are known to contribute to 634 a superficial bias in the measured BOLD signal; that is, increased BOLD with increasing 635 distance from white matter. In particular, it has been shown that the BOLD signal measured 636 using GE-EPI (i.e. T2* weighted) is confounded by macro- and micro-vasculature signals ${ }^{32,63,64}$. 
637 The macro-vasculature contribution is due to veins penetrating the grey matter and running

638 through its thickness, as well as large pial veins situated along the surface of the grey matter ${ }^{65}$.

639 This results in increased sensitivity (i.e., strong BOLD effect) but decreased spatial specificity of

640 the measured signal. The latter can be understood by the mechanics of the draining veins

641 carrying deoxygenated haemoglobin downstream from the true neuronal site of neural activation,

642 leading to a response spatially biased towards the pial surface, an effect known as superficial bias.

643 Here, we took the following approach to reduce superficial bias due to vasculature

644 contributions. First, following previous work ${ }^{66}$, we computed the temporal signal to noise ratio

645 (tSNR) for each voxel in each ROI (V1 V2, V3, V4 and IPS respectively). We used tSNR to

646 identify voxels near large veins that are expected to have large variance and low intensity signal

647 due to the local concentration of deoxygenated haemoglobin resulting in a short $\mathrm{T} 2 *$ decay time

648 (i.e., dark intensity in a T2* weighted image). We identified voxels with low tSNR, checked their

649 correspondence with voxels of lower intensities on the T2* weighted images. Second, it has been

650 shown that high t-values on a fMRI statistical map are likely to arise from large pial veins ${ }^{67,68}$.

651 Therefore, voxels with low tSNR values or t-score values above the $90^{\text {th }}$ percentile of the t-score

652 distribution obtained by the GLM described above were removed from further analysis. We used

653 these two approaches to correct the BOLD signal from confounding vasculature effects.

654 Univariate analysis. For each participant, test session, run and condition, we extracted the z-

655 scored fMRI responses between the $4^{\text {th }}$ and $8^{\text {th }}$ TR (i.e. $6.18-14.42 \mathrm{~s}$ ) after block onset. This

656 time window captured the peak of the hemodynamic responses to the visual stimuli. The

657 normalized fMRI responses were averaged across time points, blocks and runs for each condition

658 and each session. Repeated-measures ANOVA was used to test the univariate difference across

659 conditions. 
Multivariate pattern analysis. We used multivariate pattern analysis (MVPA) to decode: a)

661 trained vs. control orientation, b) untrained vs. control orientation. For each ROI and participant,

662 we calculated per voxel a t-score statistic by comparing activity for stimuli that were presented

663 left vs. right of the fixation (V1) or activity for task vs. fixation (IPS). We used this statistic to

664 rank the voxels within each ROI and selected voxels (500 for visual areas; 200 voxels for IPS)

665 with the higher t-score to include in the MVPA, as classification accuracy saturated across all

666 participants for these voxel pattern sizes in the corresponding regions (Figure S4). We used the

667 same number of voxels (i.e. 200 voxels) when comparing data between V1 and IPS and for the

668 informational connectivity analysis. This voxel selection procedure ensured that comparisons of

669 MVPA accuracy could not be confounded by varying number of voxels across participants. We

670 then extracted mean normalized fMRI responses between $4^{\text {th }}$ to $8^{\text {th }}$ TR (i.e. $6.18-14.42 \mathrm{~s}$ ) after

671 block onset for this pattern of voxels per ROI, participant and test session. We trained a linear

672 classifier using LIBSVM (http://www.csie.ntu.edu.tw/ cjlin/libsvm/) implemented in MATLAB

673 to discriminate: a) the trained from the control orientation, b) the untrained from the control

674 orientation. As both the trained and untrained orientation differed equally from the control

675 orientation $\left(\sim 55^{\circ}\right)$, we hypothesized that differences in the accuracy between these two

676 classification tasks would be due to training rather than stimulus differences. We computed

677 classification accuracy using a leave-one-run-out cross-validation. That is, we divided the data

678 set into training and test data with maximum 72 training patterns (for $n=7$ participants with 8

679 runs) and 8 patterns for the test run. We averaged the classification accuracy across folds,

680 separately for each test session. We used repeated-measures ANOVAs to assess differences in

681 classification accuracy across conditions (orientation $\times$ session). Similar to the MPI for 
682 behavioral data, we defined the MPI for decoding accuracy as (post-test accuracy - pre-test 683 accuracy) / pre-test accuracy $\times 100 \%)$.

684 Further, we performed a correlation-based pattern analysis ${ }^{37}$ to consolidate our MVPA 685 results. In the correlation-based pattern analysis, the data and voxels used were identical to those 686 used in the MVPA analysis. We divided the data set into training and test data and performed a 687 leave-one-run-out cross-validation. For each dataset, we calculated the mean response of each 688 orientation for each voxel. We then calculated the Spearman correlation across voxels and 689 transformed the correlation coefficients using Fisher's z-transform. We hypothesized that the 690 correlation coefficient would be higher for data from the training and test set that related to the 691 same orientation (i.e. trained-trained orientation) than different orientations (i.e. trained-control 692 orientation). We used the difference between the same and different orientations to index the 693 information contained in each ROI. We used repeated-measures ANOVAs to examine 694 differences across conditions (orientation $\times$ session).

695 Informational Connectivity analysis. We used Informational Connectivity (IC) to identify layers 696 that share synchronized discriminability of activity related to stimulus-specific multi-voxel 697 pattern information ${ }^{36,69,70}$. We examined intercortical IC based on shared changes (fluctuations) 698 in pattern discriminability over time, as this approach has been shown to be more sensitive than 699 univariate functional connectivity. To track the flow of multivariate information across time (i.e. 700 across blocks), we measured the fluctuations (covariance) in MVPA discriminability by 701 calculating distance information from the classification hyperplane (Figure 7A). In particular, we 702 selected 200 voxels with the higher t-score and used the same multivoxel training vs. test 703 patterns as in the MVPA analysis. For each ROI and layer, we extracted distance information for 704 the test data per block from the trained classifiers. We calculated layer-specific connectivity by 
bioRxiv preprint doi: https://doi.org/10.1101/2020.04.07.030510; this version posted May 15, 2020. The copyright holder for this preprint (which

was not certified by peer review) is the author/funder, who has granted bioRxiv a license to display the preprint in perpetuity. It is made available under aCC-BY-NC-ND 4.0 International license.

705 partial Spearman correlation between the fold-wise distance of different layers; that is, for a

706 given layer, we regressed out the distance information from other layers within each ROI. We

707 transformed the correlation coefficients using Fisher's z-transform and conducted repeated

708 measures ANOVA to compare across conditions. 


\section{References}

710 1. Gilbert, C. D., Sigman, M. \& Crist, R. E. The Neural Basis of Perceptual Learning. Neuron 31, 681-697 (2001).

712 2. Gilbert, C. D. \& Li, W. Adult Visual Cortical Plasticity. Neuron 75, 250-264 (2012).

713 3. Hooks, B. M. \& Chen, C. Circuitry Underlying Experience-Dependent Plasticity in the Mouse Visual System. Neuron 106, 21-36 (2020).

4. Sagi, D. \& Tanne, D. Perceptual learning: learning to see. Curr. Opin. Neurobiol. 4, 195199 (1994).

5. Dosher, B. \& Lu, Z.-L. Visual Perceptual Learning and Models. Annu. Rev. Vis. Sci. 3, 343-363 (2017).

6. Law, C. T. \& Gold, J. I. Shared mechanisms of perceptual learning and decision making. Top. Cogn. Sci. 2, 226-238 (2010).

7. Ahissar, M. \& Hochstein, S. Task difficulty and the specificity of perceptual learning. Nature 387, 401-406 (1997).

8. Schoups, A. A., Vogels, R. \& Orban, G. a. Human perceptual learning in identifying the oblique orientation: retinotopy, orientation specificity and monocularity. J. Physiol. 483, 797-810 (1995).

9. Schoups, A., Vogels, R., Qian, N. \& Orban, G. Practising orientation identification improves orientation coding in V1 neurons. Nature 412, 549-553 (2001).

10. Yan, Y. et al. Perceptual training continuously refines neuronal population codes in primary visual cortex. Nat. Neurosci. 17, 1380-1389 (2014).

11. Bao, M., Yang, L., Rios, C., He, B. \& Engel, S. a. Perceptual Learning Increases the Strength of the Earliest Signals in Visual Cortex. J. Neurosci. 30, 15080-15084 (2010).

12. Jehee, J. F. M., Ling, S., Swisher, J. D., Bergen, R. S. Van \& Tong, F. Perceptual learning selectively refines orientation representations in Early Visual Cortex. J. Neurosci. 32, 16747-16753 (2012).

13. Schwartz, S., Maquet, P. \& Frith, C. Neural correlates of perceptual learning : A functional MRI study of visual texture discrimination. Proc. Natl. Acad. Sci. 99, 1713717142 (2002).

14. Raiguel, S., Vogels, R., Mysore, S. G. \& Orban, G. a. Learning to see the difference specifically alters the most informative V4 neurons. J. Neurosci. 26, 6589-6602 (2006).

15. Yang, T. \& Maunsell, J. H. R. The Effect of Perceptual Learning on Neuronal Responses in Monkey Visual Area V4. J. Neurosci. 24, 1617-1626 (2004).

16. Kahnt, T., Grueschow, M., Speck, O. \& Haynes, J. D. Perceptual Learning and DecisionMaking in Human Medial Frontal Cortex. Neuron 70, 549-559 (2011).

17. Ahissar, M. \& Hochstein, S. The reverse hierarchy theory of visual perceptual learning. Trends Cogn. Sci. 8, 457-464 (2004).

18. Goense, J., Bohraus, Y. \& Logothetis, N. K. fMRI at high spatial resolution: implications for BOLD-models. Front. Comput. Neurosci. 10, (2016).

19. RockLand, K. S. \& Pandya, D. N. Laminar origins and terminations of cortical connections of the occipital lobe in the rhesus monkey. Brain Res. 179, 3-20 (1979).

20. Markov, N. T. et al. Anatomy of Hierarchy: Feedforward and Feedback Pathways in Macaque Visual Cortex. J. Comp. Neurol. 522, 225-259 (2014).

21. Larkum, M. E., Petro, L. S., Sachdev, R. N. S. \& Muckli, L. A Perspective on Cortical Layering and Layer-Spanning Neuronal Elements. Front. Neuroanat. 12, (2018). 
22. Self, M. W., van Kerkoerle, T., Goebel, R. \& Roelfsema, P. R. Benchmarking laminar fMRI: Neuronal spiking and synaptic activity during top-down and bottom-up processing in the different layers of cortex. Neuroimage 197, 806-817 (2019).

23. Gilbert, C. D. \& Wiesel, T. Clustered intrinsic connections in cat visual cortex. $J$. Neurosci. 3, 1116-1133 (1983).

24. Douglas, R. J. \& Martin, K. A. C. Recurrent neuronal circuits in the neocortex. Curr. Biol. 17, 496-500 (2007).

25. Schwabe, L. \& Obermayer, K. Adaptivity of tuning functions in a generic recurrent network model of a cortical hypercolumn. J. Neurosci. 25, 3323-3332 (2005).

26. Self, M. W., van Kerkoerle, T., Supèr, H. \& Roelfsema, P. R. Distinct roles of the cortical layers of area V1 in figure-ground segregation. Curr. Biol. 23, 2121-2129 (2013).

27. Buffalo, E. A., Fries, P., Landman, R., Buschman, T. J. \& Desimone, R. Laminar differences in gamma and alpha coherence in the ventral stream. Proc. Natl. Acad. Sci. 108, 11262-11267 (2011).

28. Martino, F. De et al. Frequency preference and attention effects across cortical depths in the human primary auditory cortex. Proc. Natl. Acad. Sci. 112, 16036-16041 (2015).

29. Lawrence, S. J. D., Norris, D. G. \& De Lange, F. P. Dissociable laminar profiles of concurrent bottom-up and top-down modulation in the human visual cortex. Elife 8, 1-17 (2019).

30. Hung, S. \& Seitz, A. R. Prolonged Training at Threshold Promotes Robust Retinotopic Specificity in Perceptual Learning. J. Neurosci. 34, 8423-8431 (2014).

31. Zhang, J. Y. et al. Rule-based learning explains visual perceptual learning and its specificity and transfer. J. Neurosci. 30, 12323-12328 (2010).

32. Uğurbil, K., Toth, L. \& Kim, D. S. How accurate is magnetic resonance imaging of brain function? Trends Neurosci. 26, 108-114 (2003).

33. Kay, K. et al. A critical assessment of data quality and venous effects in sub-millimeter fMRI. Neuroimage 189, 847-869 (2019).

34. Vizioli, L. et al. Multivoxel Pattern of Blood Oxygen Level Dependent Activity can be sensitive to stimulus specific fine scale responses. bioRxiv (2019).

35. Kok, P. et al. Selective Activation of the Deep Layers of the Human Primary Visual Cortex by Top-Down Feedback. Curr. Biol. 26, 371-376 (2016).

36. Koster, R., Chadwick, M. J., Chen, Y. \& Kumaran, D. Big-Loop Recurrence within the Hippocampal System Supports Integration of Information across Episodes. Neuron 99, 1342-1354 (2018).

37. Haxby, J. V. et al. Distributed and overlapping representations of faces and objects in ventral temporal cortex. Science (80-. ). 293, 2425-2430 (2001).

38. Chen, N. et al. Sharpened cortical tuning and enhanced cortico-cortical communication contribute to the long-term neural mechanisms of visual motion perceptual learning. Neuroimage 115, 17-29 (2015).

39. Byers, A. \& Serences, J. T. Exploring the relationship between perceptual learning and top-down attentional control. Vision Res. 74, 30-39 (2012).

40. Gilbert, C. D., Li, W. \& Piech, V. Perceptual learning and adult cortical plasticity. J. Physiol. 587, 2743-51 (2009).

41. Zhang, J., Meeson, A., Welchman, A. E. \& Kourtzi, Z. Learning alters the tuning of functional magnetic resonance imaging patterns for visual forms. J. Neurosci. 30, 1412714133 (2010). 
42. Furmanski, C. S., Schluppeck, D. \& Engel, S. A. Learning Strengthens the Response of Primary Visual Cortex to Simple Patterns. Curr. Biol. 14, 573-578 (2004).

43. Mukai, I. et al. Activations in visual and attention-related areas predict and correlate with the degree of perceptual learning. J. Neurosci. 27, 11401-11411 (2007).

44. Ringach, D. L., Shapley, R. M. \& Hawken, M. J. Orientation selectivity in macaque V1: diversity and laminar dependence. J. Neurosci. 22, 5639-5651 (2002).

45. Gau, R., Bazin, P.-L., Trampel, R., Turner, R. \& Noppeney, U. Resolving multisensory and attentional influences across cortical depth in sensory cortices. Elife 9, (2020).

46. Muckli, L. et al. Contextual Feedback to Superficial Layers of V1. Curr. Biol. 25, 26902695 (2015).

47. Chang, D. H. F., Mevorach, C., Kourtzi, Z. \& Welchman, A. E. Training transfers the limits on perception from parietal to ventral cortex. Curr. Biol. 24, 2445-2450 (2014).

48. Malach, R., Amir, Y., Harel, M. \& Grinvald, A. Relationship between intrinsic connections and functional architecture revealed by optical imaging and in vivo targeted biocytin injections in primate striate cortex. Proc. Natl Acad. Sci. USA 90, 10469-10473 (1993).

49. Teich, A. F. \& Qian, N. Learning and adaptation in a recurrent model of V1 orientation selectivity. J. Neurophysiol. 89, 2086-2100 (2003).

50. Huber, L., Uludağ, K. \& Möller, H. E. Non-BOLD contrast for laminar fMRI in humans: CBF, CBV, and CMRO2. Neuroimage 197, 742-760 (2019).

51. Watanabe, T. \& Sasaki, Y. Perceptual Learning: Toward a Comprehensive Theory. Annu. Rev. Psychol. 66, 1-25 (2015).

52. Lewis, C. M., Baldassarre, A., Committeri, G., Romani, G. L. \& Corbetta, M. Learning sculpts the spontaneous activity of the resting human brain. Proc. Natl. Acad. Sci. 106, 17558-17563 (2009).

53. Van Essen, D. C. \& Felleman, D. J. Distributed Hierarchical Processing in the Primate Cerebral Cortex. Cereb. Cortex 1, 1-47 (1991).

54. Pelli, D. G. The VideoToolbox software for visual psychophysics: transforming numbers into movies. Spat. Vis. 10, 437-442 (1997).

55. Brainard, D. H. The Psychophysics Toolbox. Spat. Vis. 10, 433-436 (1997).

56. Moeller, S. et al. Multiband multislice GE-EPI at 7 tesla, with 16-fold acceleration using partial parallel imaging with application to high spatial and temporal whole-brain FMRI. Magn. Reson. Med. 63, 1144-1153 (2010).

57. Waehnert, M. D. et al. Anatomically motivated modeling of cortical laminae. Neuroimage 93, 210-220 (2014).

58. Kemper, V. G., De Martino, F., Emmerling, T. C., Yacoub, E. \& Goebel, R. High resolution data analysis strategies for mesoscale human functional MRI at 7 and $9.4 \mathrm{~T}$. Neuroimage 164, 48-58 (2018).

59. Greve, D. N. \& Fischl, B. Accurate and robust brain image alignment using boundarybased registration. Neuroimage 48, 63-72 (2009).

60. Engel, S. a, Wandell, B. a \& Glover, G. G. Retintopic organization in human visual cortex and the spatial precision of functional MRI. Cereb. Cortex 7, 181-192 (1997).

61. Sereno, M. I. et al. Borders of Multiple Visual Areas In Humans Revealed By Functional MRI. Science (80-. ). 268, 889-893 (1995).

62. Wang, L., Mruczek, R. E. B., Arcaro, M. J. \& Kastner, S. Probabilistic maps of visual topography in human cortex. Cereb. Cortex 25, 3911-3931 (2015). 
63. Uludağ, K., Müller-Bierl, B. \& Uğurbil, K. An integrative model for neuronal activityinduced signal changes for gradient and spin echo functional imaging. Neuroimage 48, 150-165 (2009).

64. Yacoub, E., Van De Moortele, P. F., Shmuel, A. \& Uğurbil, K. Signal and noise characteristics of Hahn SE and GE BOLD fMRI at $7 \mathrm{~T}$ in humans. Neuroimage 24, 738750 (2005).

65. Duvernoy, H. M., Delon, S. \& Vannson, J. L. Cortical blood vessels of the human brain. Brain Res. Bull. 7, 519-579 (1981).

66. Olman, C. A., Inati, S. \& Heeger, D. J. The effect of large veins on spatial localization with GE BOLD at 3 T: Displacement, not blurring. Neuroimage 34, 1126-1135 (2007).

67. Polimeni, J. R., Fischl, B., Greve, D. N. \& Wald, L. L. Laminar analysis of 7 T BOLD using an imposed spatial activation pattern in human V1. Neuroimage 52, 1334-1346 (2010).

68. Kashyap, S., Ivanov, D., Havlicek, M., Poser, B. A. \& Uludağ, K. Impact of acquisition and analysis strategies on cortical depth-dependent fMRI. Neuroimage 168, 332-344 (2018).

69. Coutanche, M. N. \& Thompson-Schill, S. L. Using informational connectivity to measure the synchronous emergence of fMRI multi-voxel information across time. J. Vis. Exp. 1-7 (2014) doi:10.3791/51226.

70. Anzellotti, S. \& Coutanche, M. N. Beyond Functional Connectivity: Investigating Networks of Multivariate Representations. Trends Cogn. Sci. 22, 258-269 (2018). 


\section{Acknowledgements:}

869 We would like to thank Valentyna Chernova and Cher Zhou for help with the analysis. We are

870 grateful to Cong Yu and Sheng Li for their helpful comments and suggestions on this work. This

871 work was supported by grants to Z.K. from the Biotechnology and Biological Sciences Research

872 Council (H012508 and BB/P021255/1), the Wellcome Trust (205067/Z/16/Z) and European

873 Union's Horizon 2020 research and innovation programme under the Marie Skłodowska Curie

874 grant agreement No 840271. The 7T MRI was funded by the MRC (MR/M008983/1) and the

875 NIHR Cambridge Biomedical Research Centre. C.T.R. is funded by the Wellcome Trust and the

876 Royal Society (098436/Z/12/B).

877

878 Author contributions:

879 K.J. and Z.K. conceived the project and designed the experiments. V.K., C.R., C.T.R., G.W. and

880 R.G. developed the GE EPI protocol used in the study. K.J. and E.Z. performed the experiments.

881 K.J., E.Z, N.R.G., and A.N. developed the analysis pipeline and analyzed the data. K.J. and Z.K.

882 wrote the manuscript.

883

884 Declaration of interests:

885 The authors declare no competing interests. 


\section{Figures}

892

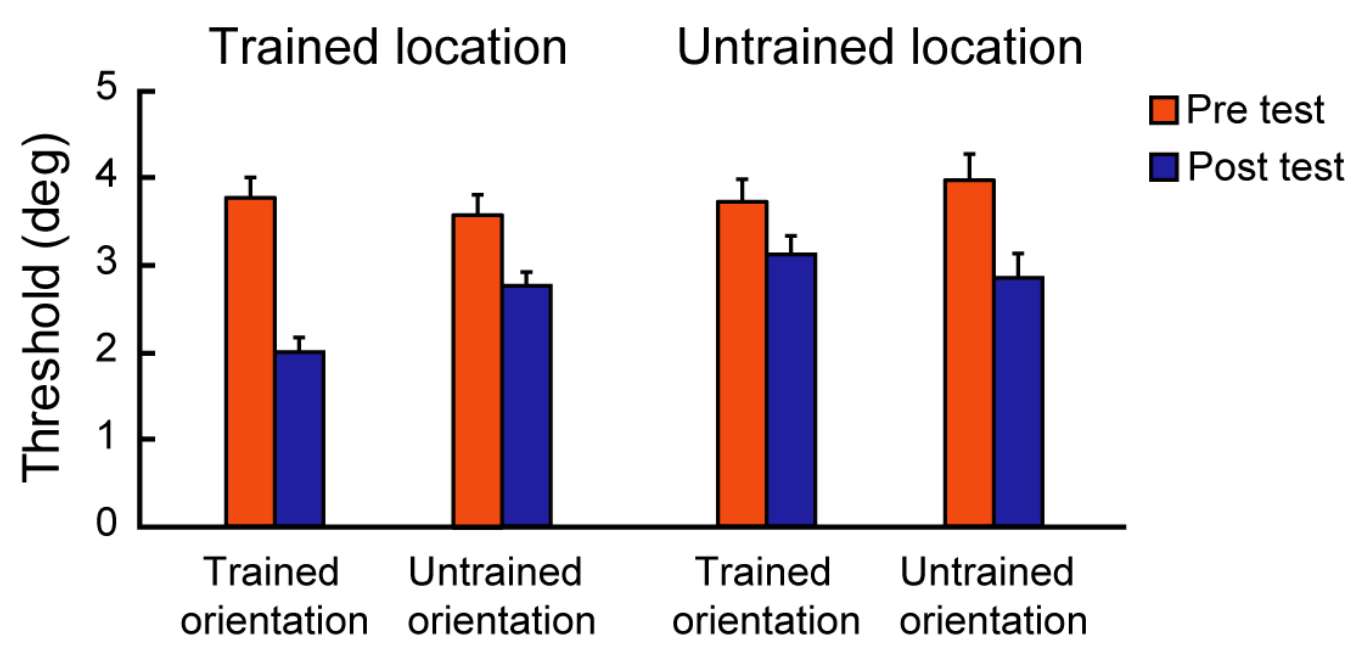

Figure S1. Behavioral results.

896 Mean performance across participants before and after training (pre-, post-test) at $\sim 79.4 \%$ threshold for

897 the trained and untrained orientations presented at the trained and untrained locations. Error bars indicate 898 standard error of the mean across participants. We observed learning specificity for the trained orientation 899 at the trained location, as indicated by a significant orientation $\times$ location $\times$ session interaction (repeated 900 measures ANOVA, $\mathrm{F}(1,12)=11.858, p=0.005)$ and a significant orientation $\times$ session interaction $901(\mathrm{~F}(1,12)=21.551, p=0.001)$ at the trained, but not the untrained $(\mathrm{F}(1,12)=3.093, p=0.104)$ location.

902 Post-hoc comparisons at the trained location showed significantly lower threshold for the trained than the 903 untrained orientation after $(\mathrm{t}(12)=-5.208, p<0.001)$, but not before $(\mathrm{t}(12)=1.264, p=0.230)$ training.

904 In contrast, no significant differences between the trained and the untrained orientations were observed at 905 the untrained location (pre-test session: $\mathrm{t}(12)=-1.203, p=0.252$, post-test session: $\mathrm{t}(12)=1.066, p=$ $906 \quad 0.308)$. 


\section{B}
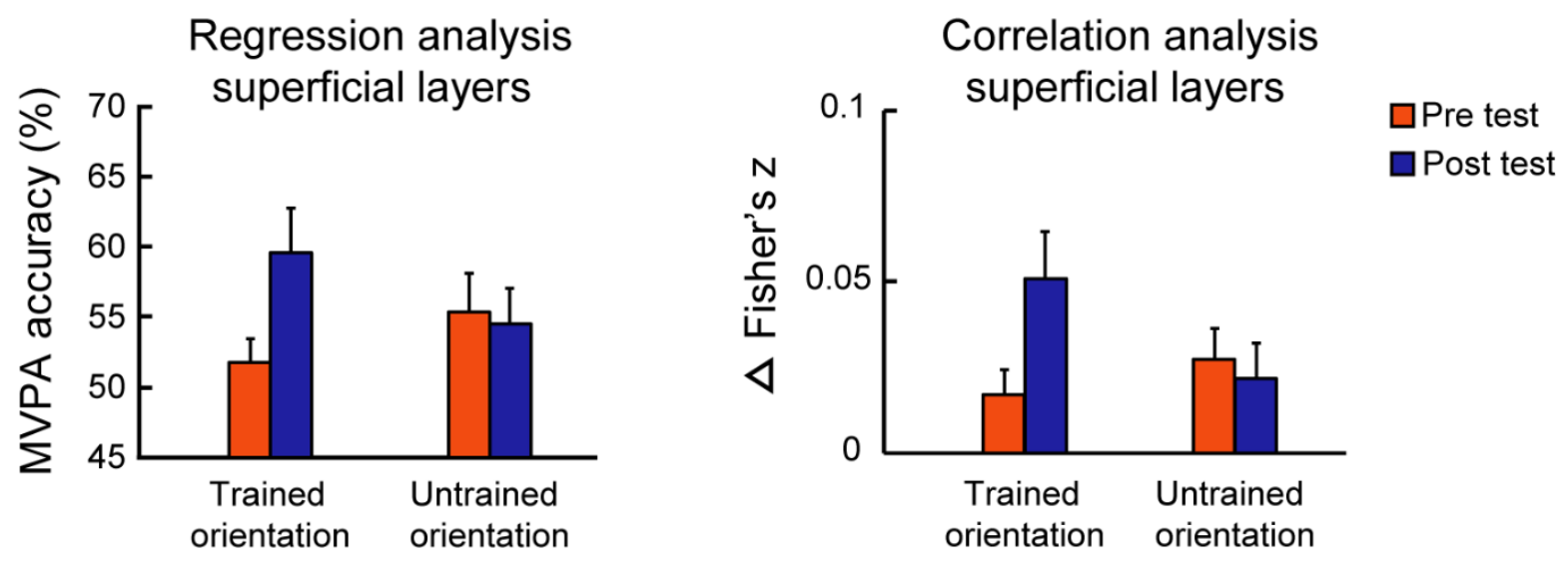

912 Figure S2. Control analyses.

913 (A) MVPA accuracy before and after training for the trained and untrained orientations presented at the

914 trained location in superficial V1 layers after regressing out the signal from the adjacent voxels in middle

915 layers. (B) Correlation-based pattern analysis. Correlation differences (correlation of mean normalized

916 BOLD across voxels for the same orientation minus normalized BOLD for different orientations) for the

917 trained and untrained orientations presented at the trained location in superficial V1 layers. Error bars

918 indicate standard error of the mean across participants. 
A

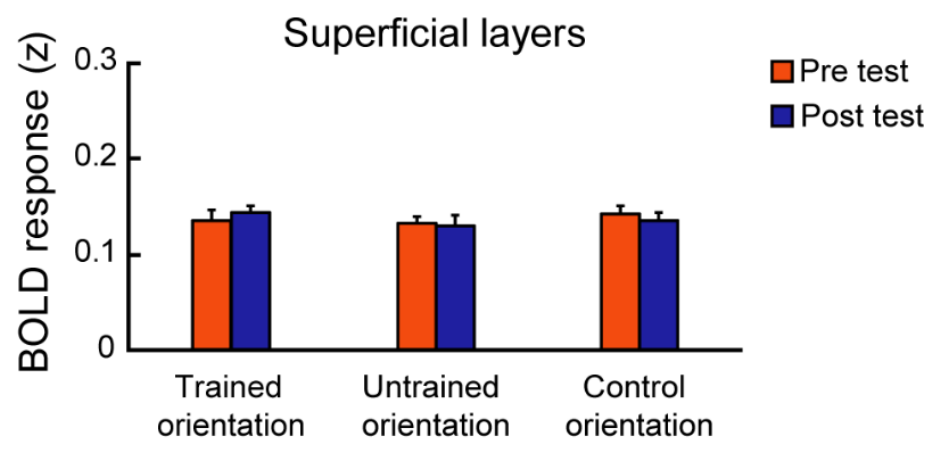

B

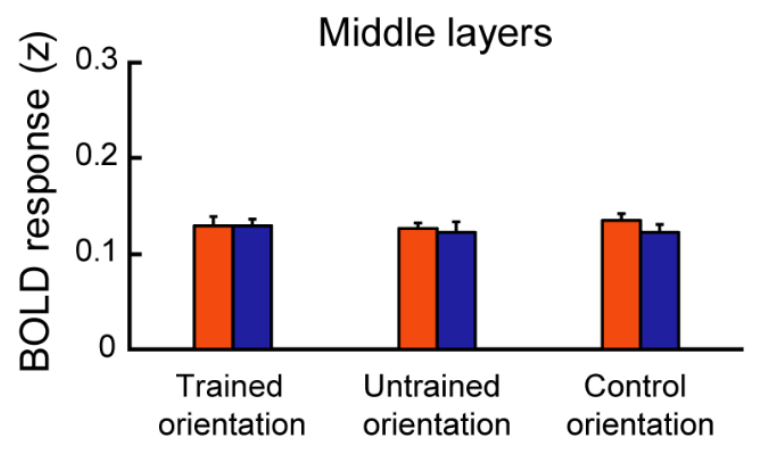

C

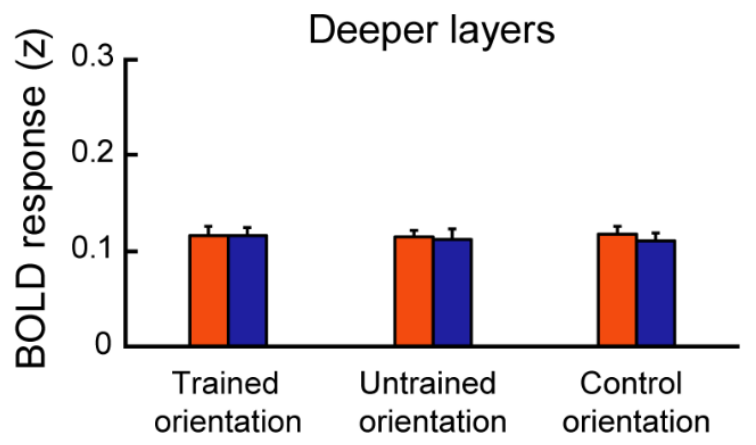

\section{Figure S3. Univariate fMRI analysis.}

924 Mean normalized BOLD before and after training for the trained, untrained and control orientations

925 presented at the trained location across V1 layers. Error bars indicate standard error of the mean across 926 participants. 


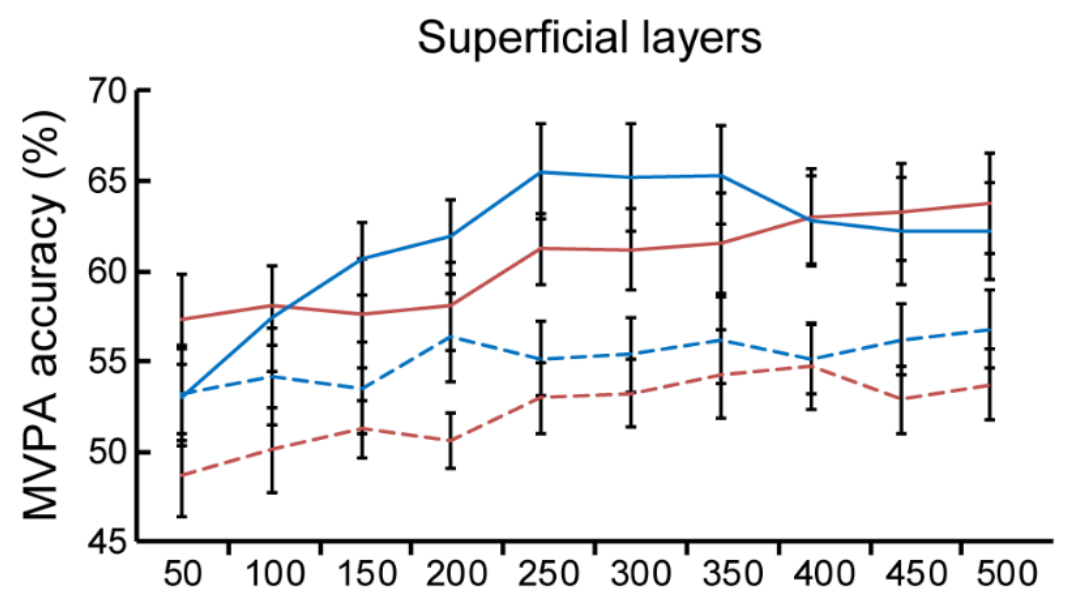

--- V1 Pre test

- V1 Post test

--- V2V3V4 Pre test V2V3V4 Post test

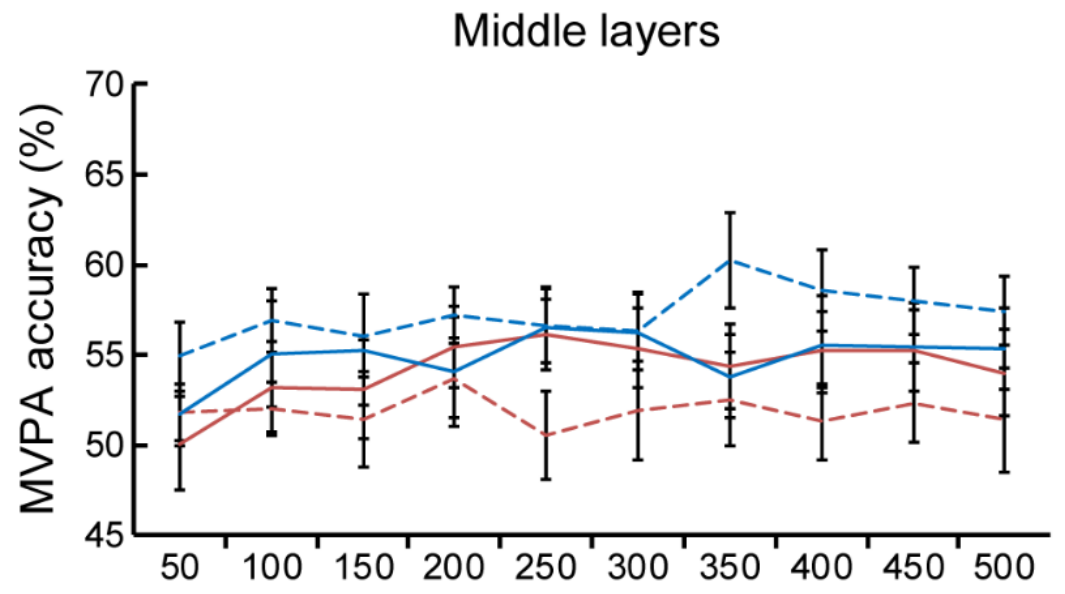

Deeper layers

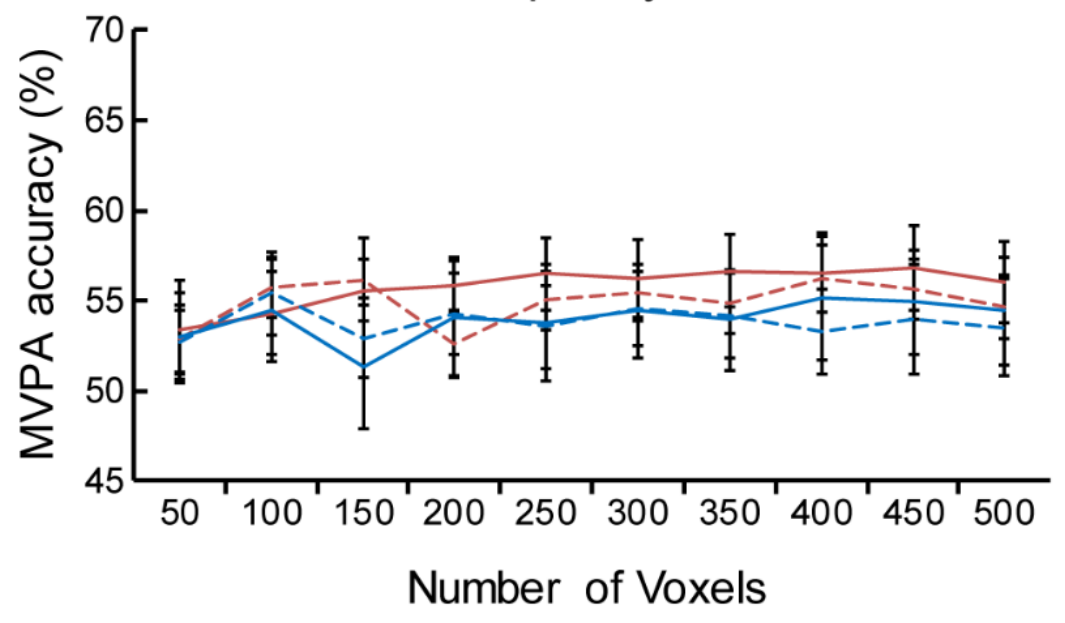




\section{Figure S4. MVPA before and after training across visual areas.}

931 MVPA accuracy before and after training for different voxel patterns (from 100-500 voxels) for the

932 trained orientation presented at the trained location across layers of V1, V2, V3, V4. We observed similar

933 learning-dependent changes in superficial layers across visual areas. Two-way repeated measures

934 ANOVAs $($ ROI $\times$ session $)$ showed a significant main effect of session for pattern size of $200(\mathrm{~F}(1,12)=$

$9357.159, p=0.020), 300(\mathrm{~F}(1,12)=7.751, p=0.017), 400(\mathrm{~F}(1,12)=6.006, p=0.031)$ voxels, and a trend

936 for pattern size of $100(\mathrm{~F}(1,12)=3.743, p=0.077)$ and $500(\mathrm{~F}(1,12)=4.587, p=0.053)$ voxels. We did

937 not observe any significant ROI $\times$ session interaction (all $p s>0.269$ ). Further, we did not observe any

938 significant differences in MVPA accuracy before vs. after training in middle nor deeper layers (all $p s>$ 939 0.539). Error bars indicate standard error of the mean across participants. 